\title{
Global Geometrical Constraints on the Shape of Proteins and Their Influence on Allosteric Regulation
}

\author{
Naoto Morikawa \\ Genocript, Zama, Japan \\ Email:nmorika@genocript.com
}

How to cite this paper: Morikawa, N. (2018) Global Geometrical Constraints on the Shape of Proteins and Their Influence on Allosteric Regulation. Applied Mathematics, 9, 1116-1155.

https://doi.org/10.4236/am.2018.910076

Received: September 14, 2018

Accepted: October 21, 2018

Published: October 24, 2018

Copyright $\odot 2018$ by author and Scientific Research Publishing Inc. This work is licensed under the Creative Commons Attribution International License (CC BY 4.0).

http://creativecommons.org/licenses/by/4.0/

\begin{abstract}
Proteins are the workhorse molecules of the cell, which are obtained by folding long chains of amino acids. Since not all shapes are obtained as a folded chain of amino acids, there should be global geometrical constraints on the shape. Moreover, since the function of a protein is largely determined by its shape, constraints on the shape should have some influence on its interaction with other proteins. In this paper, we consider global geometrical constraints on the shape of proteins. Using a mathematical toy model, in which proteins are represented as closed chains of tetrahedrons, we have identified not only global geometrical constraints on the shape of proteins, but also their influence on protein interactions. As an example, we show that a garlic-bulb like structure appears as a result of the constraints. Regarding the influence of global geometrical constraints on interactions, we consider their influence on the structural coupling of two distal sites in allosteric regulation. We then show the inseparable relationship between global geometrical constraints and protein interactions; i.e. they are different sides of the same coin. This finding could be important for the understanding of the basic mechanisms of allosteric regulation of protein functions.
\end{abstract}

\section{Keywords}

Differential Geometry, Protein Structure, Discrete Mathematics, Allosteric Regulation, Simultaneous Equations for Shape

\section{Introduction}

In this paper, we consider global geometrical constraints on the shape of proteins, using the mathematical toy model of proteins proposed in [1]. Proteins 
are the workhorse molecules of the cell, which are obtained as a complex of folded chains of amino acids. Since the function of proteins depends primarily on their shape, structural studies are essential for understanding proteins. In our approach, protein molecules are represented as a complex of closed trajectories of tetrahedrons. Then, the surface of proteins is obtained as the intersection of a pair of four-dimensional cones [2]. Interactions between proteins are defined (or mimicked) as "fusion and fission" of closed trajectories.

Previously, two types of geometrical constraints are known in the study of protein structures. One is a set of constraints on the backbone conformation due to collisions between atoms [3]. The backbone conformation is determined by torsion angle pairs $(\phi, \psi)$ along the backbone, and their allowed values are shown in the Ramachandran map [4]. The other is a set of constraints on relative distances between certain pairs of atoms, which are obtained from either physical experiments or theoretical estimates. The determination of protein structures which satisfy a set of constraints on inter-atomic distances, known as the distance geometry problem, is an important problem in structural biology [5].

In virology, another type of geometrical constraints, the symmetry of the virus structure, is also considered. Viruses are metastable macromolecular assemblies composed of the viral genome enclosed within the protein shells, called viral capsids [6]. Virus capsids are highly specific assemblies that are formed from a large number of often identical subunits. Formulated in [7] is a set of structural constraints on the subunit arrangements, using an extension of the underlying symmetry group. On the other hand, [8] finds that some viruses allow their representation as two-dimensional monohedral tilings of a bound surface, where each tile represents a subunit. Note that viral molecules consist of separeated parts. Protein molecules are obtained by folding a chain of linked parts and it is impossible to describe the shape of proteins by symmetry alone nor to describe their surface by tiling of basic subunits.

What we will consider below are global constraints on the shape of a complex of folded chains of basic blocks, such as triangles and tetrahedrons. One of the advantages of our model is the correspondence between "the shape of molecules" and "interaction between molecules". Since a protein's function is largely determined by its shape, constraints on the shape of a protein should have some influence on its interaction with other proteins. In our model, the geometrical constraints on the shape of a molecule correspond to the constraints on the interaction between three molecules, such as allosteric regulations. In the section before the conclusion, we will explain the correspondence between geometrical constraints on the shape and allosteric regulations using an example. An introduction to allosteric regulation is also given there.

Finally, Genocript (http://www.genocript.com) is the one-man bio-venture started by Naoto Morikawa in 2000 which is developing software tools for protein structure analysis. 


\section{Discrete Differential Geometry of Triangles}

Now, let us consider the case of closed trajectories of triangles to explain the basic ideas behind our approach. For detailed description, see [1] and [2].

In the following, the coordinates of points in the $N$-dimensional Eucledean space $E^{N} \quad(N=3$ or 4$)$ are represented by a monomial in $N$ indeterminates $x_{0}, x_{1}, \cdots, x_{N-1}$ for space saving purposes. For example, point $(l, m, n) \in \mathbb{Z}^{3} \subset E^{3}$ is represented by $x_{0}^{l} x_{1}^{m} x_{2}^{n}$, where $\mathbb{Z}$ denotes the set of all integers. $(0,0,0)$, $(0, m, n),(l, 0, n)$, and $(l, m, 0)$ are denoted by $1, x_{1}^{m} x_{2}^{n}, x_{0}^{1} x_{2}^{n}$, and $x_{0}^{1} x_{1}^{m}$, respectively. Let $p=x_{0}^{l} x_{1}^{m} x_{2}^{n}$. Then, points $(l+k, m, n),(l, m+k, n)$ and $(l, m, n+k)$ are represented by monomials $p x_{0}^{k}, p x_{1}^{k}$ and $p x_{2}^{k}$, respectively. Note that $x_{i} x_{j}=x_{j} x_{i}$ for all pairs of $i$ and $j$.

\subsection{Flows of Triangles}

Flows of triangles are defined using unit cubes in $E^{3}$. As shown in Figure 1(a), unit cubes are piled-up in the direction of $(-1,-1,-1)$ in $E^{3}$, where each of the three upper faces is divided into two triangles by the vertical diagonal (thick line). Then, a flow of triangles is obtained along the diagonals (Figure 1(b)). That is, the piled-up cubes form a mountain range-like structure and the vertical diagonals on its surface determine a flow of "slant" triangles on the slope.

As an example, let us consider the unit cube with the eight corner points 0 , $x_{0}, x_{0} x_{1}, x_{1}, x_{2}, x_{0} x_{2}, x_{0} x_{1} x_{2}$, and $x_{1} x_{2}$ (Figure $1(\mathrm{c})$ ). Let $P_{0}=1, P_{1}=x_{0}$, $P_{2}=x_{0} x_{1}$, and $P_{3}=x_{1}$. Then, the upper face $P_{0} P_{1} P_{2} P_{3}$ is divided into two "slant" triangles $P_{0} P_{1} P_{2}$ and $P_{0} P_{3} P_{2}$. The triangle flow goes down (or up) along the edge $P_{0} P_{2}$ at $P_{0} P_{1} P_{2}$ and $P_{0} P_{3} P_{2}$.

In the following, we give the mathematical definition of the mountain range-like structure and the associated flow of triangles.

Definition 1 (Standard Lattice) The three-dimensional standard lattice $L^{3}$ is the three-dimensional lattice generated by three vectors $(1,0,0),(0,1,0)$, and $(0,0,1)$. Using the monomial representation, $L^{3}$ is defined by

$$
L^{3}:=\left\{x_{0}{ }^{l} x_{1}{ }^{m} x_{2}{ }^{n} \mid l, m, n \in \mathbb{Z}\right\} \subset E^{3} .
$$

Let $p_{0}, p_{1}$, and $p_{2} \in L^{3}$. We denote the convex hull of $p_{0}, p_{1}$, and $p_{2}$ by $\left[p_{0}, p_{1}, p_{2}\right]$,i.e.,

$$
\left[p_{0}, p_{1}, p_{2}\right]:=\left\{p_{0}^{a} p_{1}^{b} p_{2}^{c} \mid a, b, c \in \mathbb{R}, a, b, c \geq 0, a+b+c=1\right\},
$$

where $\mathbb{R}$ is the set of all real numbers. The group of all permutations of the three-element set $\{0,1,2\}$ is denoted by $S_{y m}{ }^{3}$. For example, $\rho(0)=2$, $\rho(1)=1$, and $\rho(2)=0$ for $\rho=(02) \in \operatorname{Sym}^{3}$, where $\rho$ is written in cyclic notation.

Definition 2 (Slant Triangles) Let $a \in L^{3}$ and $\rho \in S_{y m}^{3}$. A slant triangle $a\left[x_{\rho(0)} x_{\rho(1)}\right]$ is the triangle defined by three points $\mathrm{a}, a x_{\rho(0)}$, and $a x_{\rho(0)} x_{\rho(1)}$, i.e.,

$$
a\left[x_{\rho(0)} x_{\rho(1)}\right]:=\left[a, a x_{\rho(0)}, a x_{\rho(0)} x_{\rho(1)}\right] .
$$




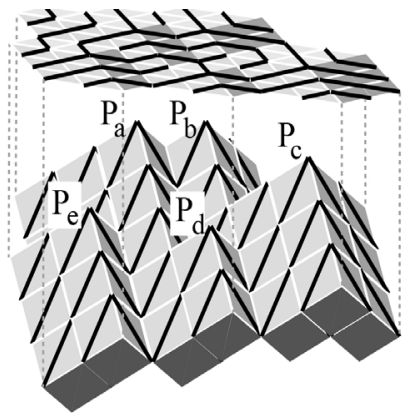

(a)

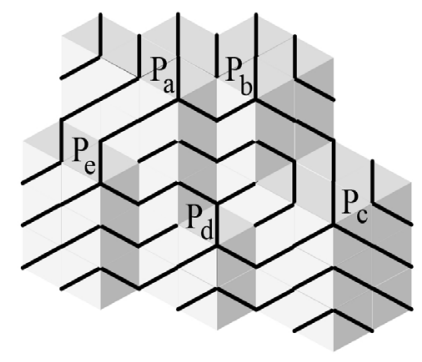

(b)

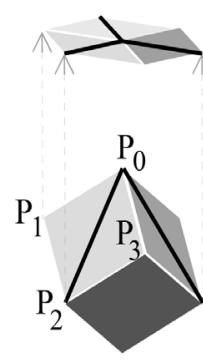

(c)

Figure 1. Flow of triangles: (a) A mountain range-like structure obtained by piling up unit cubes in the direction of $(-1,-1,-1)$, whose peaks are $P_{a}=(2,0,0), P_{b}=(1,0,1)$, $P_{c}=(-1,2,1), P_{d}=(1,3,0)$, and $P_{e}=(3,2,-1)$. The diagonal edges of "slant" triangles are drawn with thick lines. Shown above is the top view of the structure; (b) The flow of triangles determined by the mountain range-like structure of (a); (c) A unit cube and its top view (above), where $P_{0}=(0,0,0), P_{1}=(1,0,0), P_{2}=(1,1,0)$, and $P_{3}=(0,1,0)$.

The line segment joining vertex $a$ and vertex $a x_{\rho(0)} x_{\rho(1)}$ is called the diagonal edge of the slant triangle. The set $S_{2}$ of all slant triangles is defined by:

$$
S_{2}:=\left\{a\left[x_{\rho(0)} x_{\rho(1)}\right]: a \in L^{3}, \rho \in S y m^{3}\right\} .
$$

Example 1 In the case of Figure 1(c),

$$
\begin{aligned}
& P_{0} P_{1} P_{2}=\left[x_{0} x_{1}\right] \quad(a=1 \text { and } \rho=(012)), \\
& P_{0} P_{3} P_{2}=\left[x_{1} x_{0}\right] \quad(a=1 \text { and } \rho=(102)) .
\end{aligned}
$$

Their diagonal edges are the line segment $P_{0} P_{2}$.

By abuse of notation, we denote the vectors $(0,1,1),(1,0,1)$, and $(1,1,0)$ by the monomial $x_{1} x_{2}, x_{0} x_{2}$, and $x_{0} x_{1}$ respectively in the following definition.

Definition 3 (Gradient of Slant Triangles) Let $s=a\left[x_{\rho(0)} x_{\rho(1)}\right] \in S_{3}$, the gradient $D s$ of $s$ is defined by

$$
D s:=x_{\rho(0)} x_{\rho(1)} .
$$

Example 2 In the case of Example 1, the slope of $P_{0} P_{1} P_{2}$ is given by

$$
D\left(P_{0} P_{1} P_{2}\right)=D\left[x_{0} x_{1}\right]=x_{0} x_{1} \text {. }
$$

Flows of slant triangles along the diagonal edges are defined as follows.

Definition 4 (Local Trajectories of Slant Triangles) Let $s \in S_{2}$. The local trajectory of slant triangles at $s$ is a set of three consecutive slant triangles, consisting of $s$ and two adjacent slant triangles which do not include the diagonal edge of $s$. By patching "consistent" local trajectories together, we will obtain a flow of slant triangles as shown in Figure 1(a).

Let $s=a\left[x_{\rho(0)} x_{\rho(1)}\right] \in S_{2}$. The local trajectory of slant triangles at $s$ is either

$$
\left\{s_{D D}, s, s_{U U}\right\} \text { or }\left\{s_{D D}, s, s_{U D}\right\} \text { or }\left\{s_{D U}, s, s_{U U}\right\} \text { or }\left\{s_{D U}, s, s_{U D}\right\} \text {, }
$$

where 


$$
\left\{\begin{array}{l}
s_{D D}:=a x_{\rho(0)}\left[x_{\rho(1)} x_{\rho(0)}\right] \in S_{2}, \\
s_{D U}:=a x_{\rho(0)} x_{\rho(2)}^{-1}\left[x_{\rho(2)} x_{\rho(1)}\right] \in S_{2}, \\
s_{U D}:=a\left[x_{\rho(0)} x_{\rho(2)}\right] \in S_{2}, \\
s_{U U}:=a x_{\rho(1)}^{-1}\left[x_{\rho(1)} x_{\rho(0)}\right] \in S_{2}
\end{array}\right.
$$

(Figure 2(a)).

A flow of "flat" triangles is defined on the hyperplane $H_{2 D}$ using the projection $\pi_{p t}$ of $E^{3}$ onto $H_{2 D}$, where

$$
\left\{\begin{array}{l}
H_{2 D}:=\left\{x_{0}^{l} x_{1}^{m} x_{2}^{n} \mid l, m, n \in \mathbb{R}, l+m+n=0\right\} \subset E^{3}, \\
\pi_{p t}: E^{3} \rightarrow H_{2 D}, \\
\pi_{p t}\left(x_{0}^{l} x_{1}^{m} x_{2}^{n}\right):=x_{0}^{(2 l-m-n) / 3} x_{1}^{(-l+2 m-n) / 3} x_{2}^{(-l-m+2 n) / 3} .
\end{array}\right.
$$

Definition 5 (Flat Triangles) Let $s=a\left[x_{\rho(0)} x_{\rho(1)}\right] \in S_{2}$. The projection $\pi_{H}$ of s on $H_{2 D}$ is defined by

$$
\pi_{H}(s):=\left[\pi_{p t}(a), \pi_{p t}\left(a x_{\rho(0)}\right), \pi_{p t}\left(a x_{\rho(0)} x_{\rho(1)}\right)\right] \subset H_{2 D} .
$$

$\pi_{H}(s)$ is called a flat triangle. The line segment joining $\pi_{p t}(a)$ and $\pi_{p t}\left(a x_{\rho(0)} x_{\rho(1)}\right)$ is called the diagonal edge of the flat triangle (Figure $1(\mathrm{c})$ ). The set $B_{2}$ of all flat triangles on $H_{2 D}$ is defined by:

$$
B_{2}:=\left\{\pi_{H}(s) \mid s \in S_{2}\right\} .
$$

Remark $\pi_{H}(s)$ is a projection of triangles and $\pi_{p t}(p)$ is a projection of points.

By projecting slant triangles onto $H_{2 D}$, we obtain a two-dimensional flow of flat triangles on $H_{2 D}$.

Definition 6 (Local Trajectories of Flat Triangles) Let $t \in B_{2}$. The local trajectory of flat triangles at $\mathrm{t}$ is a projection image of a local trajectory of slant triangles at $s \in S_{2}$ by $\pi_{H}$, where $\pi_{H}(s)=t$. That is, there exists some $s \in S_{2}$ such that $\pi_{H}(s)=t$ and the local trajectory at $\mathrm{s}$ is given by either

$$
\begin{aligned}
& \left\{\pi_{H}\left(s_{D D}\right), \pi_{H}(s), \pi_{H}\left(s_{U U}\right)\right\} \text { or }\left\{\pi_{H}\left(s_{D D}\right), \pi_{H}(s), \pi_{H}\left(s_{U D}\right)\right\} \\
& \text { or }\left\{\pi_{H}\left(s_{D U}\right), \pi_{H}(s), \pi_{H}\left(s_{U U}\right)\right\} \text { or }\left\{\pi_{H}\left(s_{D U}\right), \pi_{H}(s), \pi_{H}\left(s_{U D}\right)\right\},
\end{aligned}
$$

where the definition of $s_{D D}, s_{D U}, s_{U D}$, and $s_{U U}$ are given above (immediately after Definition 4). Note that $\pi_{H}\left(s_{D D}\right)=\pi_{H}\left(s_{D U}\right)$ and $\pi_{H}\left(s_{U D}\right)=\pi_{H}\left(s_{U U}\right)$ (Figure 2(a)). By patching “consistent" local trajectories together, we will obtain a flow of flat triangles as shown in Figure 1(a).

\subsection{Vector Fields of Triangles}

As shown in Figure 1(a), a mountain range-like structure induces a flow of triangles on $B_{2}$. We can define a "tangent space" structure on the space $B_{2}$ of flat triangles, where each flat triangle assume one of the three gradient vectors $x_{1} x_{2}, x_{0} x_{2}$, and $x_{0} x_{1}$. 


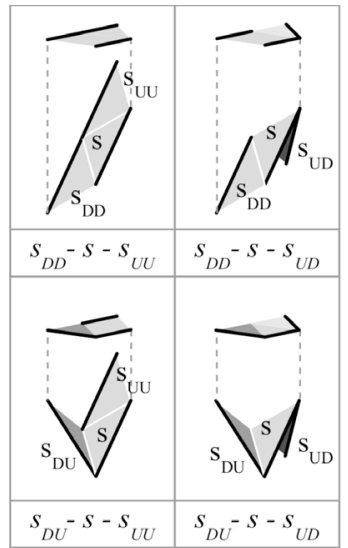

(a)

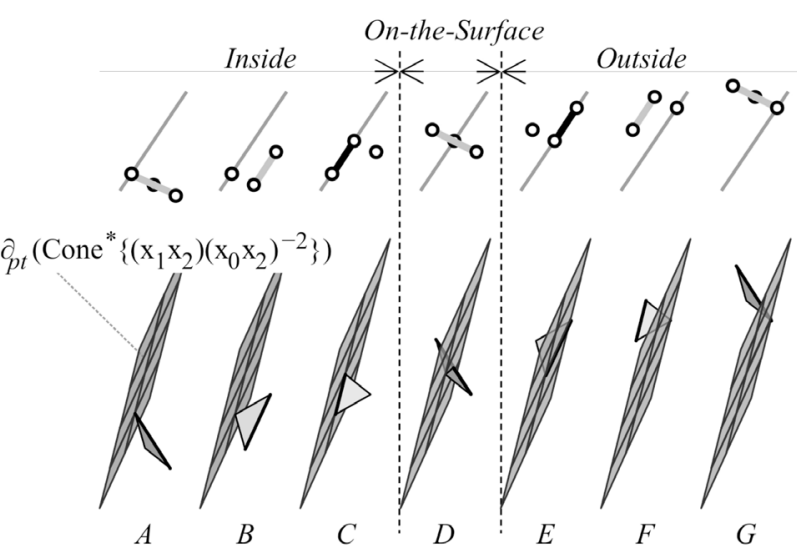

(b)

Figure 2. Slant triangles: (a) The four local trajectories of slant triangles at $s \in B_{2}$. Shown above is the top view of the trajectory; (b) Positional relationship of the slant triangles of $\pi_{H}^{-1}\left(\left[x_{1} x_{2}\right]\right)$ and the 2-face $\left\{\left(x_{1} x_{2}\right)\left(x_{0} x_{2}\right)^{m-2}\left(x_{0} x_{1}\right)^{n} \mid 0 \leq m, n \in \mathbb{Z}\right\}$ of the cotangent cone Cone $^{*}\left\{\left(x_{1} x_{2}\right)\left(x_{0} x_{2}\right)^{-2}\right\}$, where slant triangles shown are $\mathrm{A}: x_{0}^{2} x_{1}^{2} x_{2}^{2}\left[x_{1} x_{2}\right], \mathrm{B}$ : $x_{0} x_{1}^{2} x_{2}^{2}\left[x_{0} x_{1}\right], \mathrm{C}: x_{0} x_{1}^{2} x_{2}\left[x_{2} x_{0}\right], \mathrm{D}: x_{0} x_{1} x_{2}\left[x_{1} x_{2}\right], \mathrm{E}: x_{1} x_{2}\left[x_{0} x_{1}\right], \mathrm{F}: x_{1}\left[x_{2} x_{0}\right]$, and $\mathrm{G}:$ $\left[x_{1} x_{2}\right]$. Shown above is a schematic diagram of the relationship between the three vertices of a slant triangle and a 2-face of $\operatorname{Cone}^{*}\left\{\left(x_{1} x_{2}\right)\left(x_{0} x_{2}\right)^{-2}\right\}$. In the diagram, the diagonal edges of slant triangles are drawn with thick line, where the diagonal edges on the 2 -face are colored black and the others are colored grey. Note that all slant triangles are projected onto the same flat triangle $\pi_{H}\left(\left[x_{1} x_{2}\right]\right)$ by $\pi_{H}$. Triangles $\mathrm{A}, \mathrm{B}$, and $\mathrm{C}$ are included in Cone $^{*}\left\{\left(x_{1} x_{2}\right)\left(x_{0} x_{2}\right)^{-2}\right\}$. Triangle D intersects the 2 -face of the cotangent cone. Triangles E, F, and G are located outside the cotangent cone.

Definition 7 (Tangent Space) The tangent space $T B_{2}$ on $B_{2}$ is defined by

$$
\left\{\begin{array}{l}
T B_{2}:=B_{2} \times\left\{x_{1} x_{2}, x_{0} x_{2}, x_{0} x_{1}\right\}, \\
\pi: T B_{2} \rightarrow B_{2}, \quad \pi\left(\left(t, x_{i} x_{j}\right)\right):=t .
\end{array}\right.
$$

Let $t \in B_{2}$. The tangent space at $t$ is denoted by $T B_{2}[t]$. Note that there exists a one-to-one correspondence

$$
T B_{2}[t] \sim\left\{x_{1} x_{2}, x_{0} x_{2}, x_{0} x_{1}\right\} .
$$

An inverse function of the projection $\pi$, i.e., a vector field of $T B_{2}$ on $B_{2}$, is induced by a mountain range-like structure consisting of piled-up unit cubes.

Definition 8 (Tangent Cones) Let $A \subset L^{3}$, the three-dimensional tangent cone Cone $A$ is defined by

$$
\text { Cone } A:=\left\{a x_{0}^{l} x_{1}^{m} x_{2}^{n} \mid a \in A, 0 \leq l, m, n \in \mathbb{Z}\right\} \subset L^{3} .
$$

Roughly speaking, Cone $A$ is the triangular cone whose top vertices are given by $A \subset L^{3}$. The set of all the top vertices of a tangent cone $c$ is denoted by top $(c)$. In general, top $($ Cone $A) \subset A$.

Definition $9\left(\partial_{t} \operatorname{top}(c)\right)$ Let $c$ be a three-dimensional tangent cone. The peaks 
on the boundary of $c$ is defined by

$$
\begin{aligned}
\partial_{t} \text { top }(c):= & \left\{p \in \operatorname{top}(c) \mid \exists i \in\{0,1,2\} \text { s.t. } p\left(x_{i}\right)^{N} \notin \text { Cone }(\text { top }(c) \backslash\{p\})\right. \\
& \text { for } \forall N \in \mathbb{N}\},
\end{aligned}
$$

where $\mathbb{N}$ denotes the set of all natural numbers.

Example 3 Let $c$ be the tangent cone corresponds to the mountain range-like structure of Figure 1(a). Then,

$$
\left\{\begin{array}{l}
c=\text { Cone }\left\{x_{0}^{2}, x_{0} x_{2}, x_{0}^{-1} x_{1}^{2} x_{2}, x_{0} x_{1}^{3}, x_{0}^{3} x_{1}^{2} x_{2}^{-1}\right\}, \\
\operatorname{top}(c)=\partial_{t} t o p(c)=\left\{x_{0}^{2}, x_{0} x_{2}, x_{0}^{-1} x_{1}^{2} x_{2}, x_{0} x_{1}^{3}, x_{0}^{3} x_{1}^{2} x_{2}^{-1}\right\} .
\end{array}\right.
$$

Definition $10\left(\partial_{p t}(c)\right)$ Let $c$ be a three-dimensional tangent cone. The surface lattice points $\partial_{p t}(c)$ of $c$ is the set of all the $L^{3}$ lattice points on the surface of $c$, i.e.,

$$
\begin{aligned}
\partial_{p t}(c):= & \left\{a x_{i}^{l} x_{j}^{m} \mid a \in \operatorname{top}(c),\{i, j\} \subset\{0,1,2\}, 0 \leq l, m \in \mathbb{Z}\right. \text { s.t. } \\
& \left.a x_{i}^{l} x_{j}^{m} \notin \text { Cone }\left\{b x_{0} x_{1} x_{2}\right\} \text { for } \forall b \in \operatorname{top}(c)\right\} \subset L^{3} .
\end{aligned}
$$

Example 4 The surface lattice points of the three-dimensional tangent cone Cone $\{1\}$ are given by

$$
\partial_{p t}(\text { Cone }\{1\})=U_{l=0} \cup U_{m=0} \cup U_{n=0} \subset L^{3},
$$

where

$$
\left\{\begin{array}{l}
U_{l=0}:=\left\{x_{1}^{m} x_{2}^{n} \mid 0 \leq m, n \in \mathbb{Z}\right\}, \\
U_{m=0}:=\left\{x_{0}^{l} x_{2}^{n} \mid 0 \leq l, n \in \mathbb{Z}\right\}, \\
U_{n=0}:=\left\{x_{0}^{l} x_{1}^{m} \mid 0 \leq l, m \in \mathbb{Z}\right\} .
\end{array}\right.
$$

That is, $\partial_{p t}($ Cone $\{1\})$ is the union of the three 2 -faces of Cone $\{1\}$.

Definition $11\left(d_{S} c\right)$ Let $c$ be a three-dimensional tangent cone. Then, $d_{S} c$ is the set of all the slant triangles on the surface of $c$, i.e.

$$
d_{S} c:=\left\{s \in S_{2} \mid \text { all the vertices of } s \text { are included in } \partial_{p t}(c)\right\} .
$$

Definition 12 (Vector Fields) Let $c$ be a three-dimensional tangent cone. The vector field $V_{c}$ induced by $c$ on $B_{2}$ is defined by

$$
V_{c}(t):=D s \quad\left(t \in B_{2}\right) \text {, }
$$

where $s \in d_{S} c$ such that $t=\pi_{H}(s)$ (Note that $s$ is uniquely determined for each $t \in B_{2}$ ). The flow of triangles determined by $V_{c}$ is called the flow of triangles induced by $c$.

Example 5 In the case of Figure 1(c),

$$
V_{c}\left(\pi\left(\left[x_{0} x_{1}\right]\right)\right)=D\left[x_{0} x_{1}\right]=x_{0} x_{1},
$$

where $c=$ Cone $\{1\} \quad$ (Recall that $P_{0} P_{1} P_{2}=\left[x_{0} x_{1}\right]$ ).

\subsection{Contour of Closed Trajectories of Triangles}

The ridge lines of tangent cones are given by three vectors $(1,0,0),(0,1,0)$, 
and $(0,0,1)$. To compute the contour of the region of $H_{2 D}$ swept by a set of closed trajectories of flat triangles, we will consider another type of triangular cones whose ridge lines are given by the slopes of slant triangles, i.e., $(0,1,1)$, $(1,0,1)$, and $(1,1,0)$.

Definition 13 (Conjugate Lattice) The conjugate lattice $L^{* 3}$ is the three-dimensional lattice generated by three vectors $(0,1,1),(1,0,1)$, and $(1,1,0)$. Using the monomial representation, $L^{* 3}$ is given by

$$
L^{* 3}:=\left\{x_{0}^{m+n} x_{1}^{l+n} x_{2}^{l+m} \mid l, m, n \in \mathbb{Z}\right\} \subset L^{3} .
$$

Definition 14 (Cotangent Cones) Let $A \subset L^{* 3}$. The three-dimensional cotangent cone $\operatorname{Cone}^{*} A$ is defined by

$$
\text { Cone }^{*} A:=\left\{a\left(x_{1} x_{2}\right)^{l}\left(x_{0} x_{2}\right)^{m}\left(x_{0} x_{1}\right)^{n} \mid a \in A, 0 \leq l, m, n \in \mathbb{Z}\right\} \subset L^{* 3} .
$$

We denote the top vertices of a cotangent cone $c$ by top $(c)$. Note that we define cotangent cones only for $A \subset L^{* 3}$.

For a given cotangent cone, we can put a "roof" on the cone.

Definition 15 (Cotangent Roofs) Let $A \subset L^{* 3}$. The three-dimensional cotangent roof $\operatorname{Roof}^{*} A$ is defined by

$$
\begin{aligned}
\operatorname{Roof}^{*} A:= & \left\{p \in L^{* 3} \mid \exists N \in \mathbb{Z} \text { s.t. } N>0 \text { and } p\left(x_{1} x_{2}\right)^{N},\right. \\
& \left.p\left(x_{0} x_{2}\right)^{N}, p\left(x_{0} x_{1}\right)^{N} \in \text { Cone }^{*} A\right\} .
\end{aligned}
$$

Roughly speaking, $\operatorname{Roof}^{*} A$ is obtained by putting as many unit cubes of $L^{* 3}$ as possible on $\operatorname{Cone}^{*} A$.

Example 6 In the case of Figure 3(a),

$$
\begin{aligned}
& \operatorname{Roof}^{*} \partial_{t} \operatorname{top}\left(c_{0}\right) \\
& =\operatorname{Roof}^{*}\left\{x_{0}{ }^{2}, x_{0} x_{2}, x_{0}^{-1} x_{1}^{2} x_{2}, x_{0} x_{1}^{3}, x_{0}^{3} x_{1}^{2} x_{2}^{-1}\right\} \\
& =\operatorname{Cone}^{*}\left\{x_{0} x_{2}^{-1}, 1, x_{0}^{-1} x_{1}\right\} .
\end{aligned}
$$

Definition $16\left(\partial_{p t}(w)\right)$. Let $w$ be a three-dimensional cotangent cone. The surface lattice points $\partial_{p t}(w)$ of $w$ is the set of all the $L^{* 3}$ lattice points on the surface of $w$, i.e.,

$$
\begin{aligned}
\partial_{p t}(w):=\left\{a\left(e_{3} / x_{i}\right)^{l}\left(e_{3} / x_{j}\right)^{m} \mid a \in \operatorname{top}(w),\{i, j\} \subset\{0,1,2\}, 0 \leq l, m \in \mathbb{Z},\right. \\
\\
\left.a\left(e_{3} / x_{i}\right)^{l}\left(e_{3} / x_{j}\right)^{m} \notin \operatorname{Cone}^{*}\left\{b e_{3}^{2}\right\} \text { for } \forall b \in \operatorname{top}(w)\right\} \subset L^{* 3},
\end{aligned}
$$

where $e_{3}:=x_{0} x_{1} x_{2} \quad$ (For example, $e_{3} / x_{1}=x_{0} x_{2}$ and $\left.e_{3}^{2}=x_{0}^{2} x_{1}^{2} x_{2}^{2}\right)$.

Example 7 The surface lattice points of the three-dimensional cotangent cone Cone $^{*}\{1\}$ are given by

$$
\partial_{p t}\left(\operatorname{Cone}^{*}\{1\}\right)=V_{l=0} \cup V_{m=0} \cup V_{n=0} \subset L^{* 3},
$$

where

$$
\left\{\begin{array}{l}
V_{l=0}:=\left\{\left(x_{0} x_{2}\right)^{m}\left(x_{0} x_{1}\right)^{n} \mid 0 \leq m, n \in \mathbb{Z}\right\}, \\
V_{m=0}:=\left\{\left(x_{1} x_{2}\right)^{l}\left(x_{0} x_{1}\right)^{n} \mid 0 \leq l, n \in \mathbb{Z}\right\}, \\
V_{n=0}:=\left\{\left(x_{1} x_{2}\right)^{l}\left(x_{0} x_{2}\right)^{m} \mid 0 \leq l, m \in \mathbb{Z}\right\} .
\end{array}\right.
$$




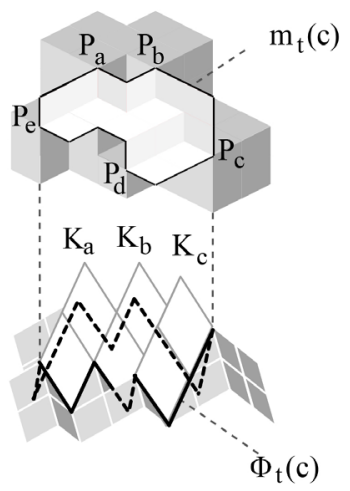

(a)

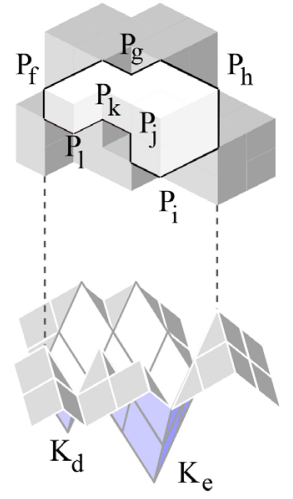

(b)

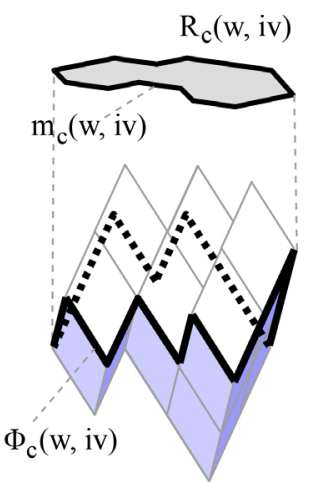

(c)

Figure 3. The closed trajectory of Figure 1 (a) and the associated contour pair: (a) Tangent cone $c_{0}=$ Cone $\left\{P_{a}, P_{b}, P_{c}, P_{d}, P_{e}\right\}$ and the associated cotangent roof $w_{c}\left(c_{0}\right)=$ Cone $^{*}\left\{K_{a}, K_{b}, K_{c}\right\}$; (b) Inverted cotangent roof

IRoof $^{*} \phi_{t}\left(c_{0}\right)=$ ICone $^{*}\left\{K_{d}, K_{e}\right\}$; (c) The region $R_{c}(w, i v)$ for the contour pair $(w, i v)=\left(\right.$ Cone $\left.^{*}\left\{K_{a}, K_{b}, K_{c}\right\}, I \operatorname{Cone}^{*}\left\{K_{d}, K_{e}\right\}\right)$ associated with $c_{0}$. In the figure, $P_{a}=x_{0}^{2}, \quad P_{b}=x_{0} x_{2}, \quad P_{c}=x_{0}^{-1} x_{1}^{2} x_{2}, \quad P_{d}=x_{0} x_{1}^{3}, \quad P_{e}=x_{0}^{3} x_{1}^{2} x_{2}^{-1}, P_{f}=x_{0}^{4} x_{1}^{2}, \quad P_{g}=x_{0}^{2} x_{1} x_{2}$, $P_{h}=x_{0} x_{1}^{2} x_{2}^{3}, \quad P_{i}=x_{0} x_{1}^{4} x_{2}, \quad P_{j}=x_{0}^{2} x_{1}^{3} x_{2}, \quad P_{k}=x_{0}^{2} x_{1}^{2} ， P_{l}=x_{0}^{3} x_{1}^{3} ， K_{a}=x_{0} x_{2}^{-1} ， K_{b}=1$, $K_{c}=x_{0}^{-1} x_{1}, K_{d}=x_{0}^{4} x_{1}^{3} x_{2}$, and $K_{e}=x_{0}^{3} x_{1}^{4} x_{2}^{3}$.

Let $c$ be a tangent cone and $w$ be a cotangent cone. Then, we can divide all the slant triangles of the flow induced by $c$ into three groups: 1 ) inside $w, 2$ ) outside $w$, and 3 ) on the surface of $w$ (Figure 2(b)). In particular, we can compute the contour of closed trajectories induced by a tangent cone using a cotangent cone as shown below.

Definition $17\left(w_{c}\left(c_{0}\right)\right)$. Let $c_{0}$ be a three-dimensional tangent cone. Suppose that $\partial_{t}$ top $\left(c_{0}\right) \subset L^{* 3}$. The three-dimensional cotangent roof $w_{c}\left(c_{0}\right)$ associated with $c_{0}$ is defined by

$$
w_{c}\left(c_{0}\right):=\operatorname{Roof}^{*} \partial_{t} \operatorname{top}\left(c_{0}\right) .
$$

Definition $18\left(\phi_{t}\left(c_{0}\right)\right)$. Let $c_{0}$ be a three-dimensional tangent cone. Suppose that $\partial_{t}$ top $\left(c_{0}\right) \subset L^{* 3}$. The contour vertices $\phi_{t}\left(c_{0}\right)$ with respect to $c_{0}$ is defined by

$$
\phi_{t}\left(c_{0}\right):=\partial_{p t}\left(c_{0}\right) \cap \partial_{p t}\left(w_{c}\left(c_{0}\right)\right) \subset L^{* 3} .
$$

That is, $\phi_{t}\left(c_{0}\right)$ is the set of $L^{* 3}$ lattice points on the intersection of the surface of $c_{0}$ and the surface of $w_{c}\left(c_{0}\right)$.

Definition $19\left(\Phi_{t}\left(c_{0}\right)\right)$. Let $c_{0}$ be a three-dimensional tangent cone. Suppose that $\partial_{t}$ top $\left(c_{0}\right) \subset L^{* 3}$. Let $\Phi_{t}\left(c_{0}\right) \subset E^{3}$ be the polygonal line obtained by joining the adjacent $L^{* 3}$ lattice points of $\phi_{t}\left(c_{0}\right)$. Since all the points of $\phi_{t}\left(c_{0}\right)$ are on the surface of $w_{c}\left(c_{0}\right)$, the points of $\phi_{t}\left(c_{0}\right)$ are connected along the surface of the associated cotangent roof. Note that $\Phi_{t}\left(c_{0}\right)$ forms a closed polygonal line if top $\left(c_{0}\right)$ is finite (Figure $3(\mathrm{a})$ ). 
Definition $20\left(m_{t}\left(c_{0}\right)\right)$. Let $c_{0}$ be a three-dimensional tangent cone. The one-dimensional surface mesh $m_{t}\left(c_{0}\right)$ with respect to $c_{0}$ is defined by

$$
m_{t}\left(c_{0}\right):=\pi_{p t}\left(\Phi_{t}\left(c_{0}\right)\right) \subset H_{2 D} .
$$

We also call $m_{t}\left(c_{0}\right)$ the (one-dimensional) contour with respect to $c_{0}$.

Definition 21 Let $c_{0}$ be a three-dimensional tangent cone. Set

$$
\left\{\begin{array}{l}
I N_{t}\left(c_{0}\right):=\left\{s \in d_{S} c_{0} \mid s \text { is contained inside } w_{c}\left(c_{0}\right)\right\}, \\
O U T_{t}\left(c_{0}\right):=\left\{s \in d_{S} c_{0} \mid s \text { is contained outside } w_{c}\left(c_{0}\right)\right\}, \\
B D_{t}\left(c_{0}\right):=\left\{s \in d_{S} c_{0} \mid s \text { intersects with the surface of } w_{c}\left(c_{0}\right)\right\} .
\end{array}\right.
$$

Theorem 1 Let $c$ be a three-dimensional tangent cone. Suppose that top $(c)$ is finite and $\partial_{t} \operatorname{top}(c) \subset L^{* 3}$. Then, $w_{c}(c)$ divides all the slant triangles of $d_{S} c$ (i.e., slant triangles on the surface of $c$ ) into two groups: inside the roof and outside the roof. That is, $B D_{t}(c)=\varnothing$ and $\pi_{H}\left(I N_{t}(c)\right)$ exactly corresponds to the region swept by all the closed trajectories of $V_{c}$.

Proof. $\Phi_{t}(c)$ forms a closed polygonal line because $\phi_{t}(c)$ contains only a finite number of points. Note that $\Phi_{t}(c)$ consists of the diagonal edges of slant triangles on the surface of $c$. Since flows of slant triangles go along the diagonal edge at each slant triangle, there is no slant triangle crossing $\Phi_{t}(c)$. That is, $\Phi_{t}(c)$ divides the flow of the slant triangles on the surface of $c$ into two parts: the inside $\Phi_{t}(c)$ and the outside $\Phi_{t}(c)$.

Definition $22\left(R_{t}(c)\right)$. Let $c$ be a three-dimensional tangent cone. Let $V_{c}$ be the vector field induced by $c$. We define the region $R_{t}(c)$ of $H_{2 D}$ by

$$
R_{t}(c):=\bigcup_{s \in N_{t}(c)} \pi_{H}(s) \subset H_{2 D} .
$$

By Theorem 1, $R_{t}(c)$ corresponds to the region of $H_{2 D}$ swept by all the closed trajectories of $V_{c}$ if $\operatorname{top}(c)$ is finite and $\partial_{t} \operatorname{top}(c) \subset L^{* 3}$.

We can compute the contour of $R_{t}(c)$ instantly.

Corollary 1 ("Contour" of $R_{t}(c)$ ). Let $c$ a three-dimensional tangent cone. Suppose that $\operatorname{top}(c)$ is finite and $\partial_{t} t o p(c) \subset L^{* 3}$. Then, $m_{t}(c)$ gives the "contour" of $R_{t}(c)$.

Proof. It follows immediately from the theorem.

Example 8 In the case of Figure 3(a), the closed polygonal line $m_{t}\left(c_{0}\right)$ consists of 12 vertices and 12 line segments, where

$$
\left\{\begin{array}{l}
c_{0}=\text { Cone }\left\{P_{a}, P_{b}, P_{c}, P_{d}, P_{e}\right\}, \\
w_{c}\left(c_{0}\right)=\text { Cone }^{*}\left\{K_{a}, K_{b}, K_{c}\right\}, \\
\phi_{t}\left(c_{0}\right)=\left\{P_{a}, P_{g}, P_{b}, P_{b h}, P_{h}, P_{h c}, P_{c}, P_{c i}, P_{i}, P_{d}, P_{j}, P_{k}, P_{l}, P_{c}, P_{f}, P_{f a}\right\},
\end{array}\right.
$$

and $P_{b h}=x_{0} x_{1} x_{2}^{2}, P_{h c}=x_{1}^{2} x_{2}, P_{c i}=x_{1}^{3} x_{2}$, and $P_{f a}=x_{0}^{3} x_{1}$.

\section{4...Constraints on the Contour of Closed Trajectories}

Let $c$ be a three-dimensional tangent cone. We have computed the contour of $R_{t}(c)$ using $c$ and the associated cotangent roof $w_{c}(c)$ (the upper row of 
Figure 4). Now, we will compute regions of $H_{2 D}$ without using tangent cones (the lower row of Figure 4).

Definition 23 (Inverted Cotangent Cones) Let $A \subset L^{* 3}$. The three-dimensional inverted cotangent cone $\operatorname{ICone}^{*} A$ is defined by

$$
\text { ICone }^{*} A:=\left\{a\left(x_{1} x_{2}\right)^{l}\left(x_{0} x_{2}\right)^{m}\left(x_{0} x_{1}\right)^{n} \mid a \in A, 0 \geq l, m, n \in \mathbb{Z}\right\} \subset L^{* 3} .
$$

We denote the top vertices of an inverted cotangent cone iv by itop $(i v)$.

Definition 24 (Inverted Cotangent Roofs) Let $A \subset L^{* 3}$. The three-dimensional inverted cotangent roof $\operatorname{IRoof}^{*} A$ is defined by

$$
\begin{gathered}
\operatorname{IRoof}^{*} A:=\left\{p \in L^{* 3} \mid \exists N \in \mathbb{Z} \text { s.t. } N<0 \text { and } p\left(x_{1} x_{2}\right)^{N},\right. \\
\left.p\left(x_{0} x_{2}\right)^{N}, p\left(x_{0} x_{1}\right)^{N} \in \text { ICone }^{*} A\right\} .
\end{gathered}
$$

Example 9 In the case of Figure 3(b),

$$
\begin{aligned}
& \operatorname{IRoof}^{*} \phi_{t}(c) \\
& =\operatorname{IRoof}^{*}\left\{x_{0}^{4} x_{1}^{2}, x_{0} x_{1}^{2} x_{2}^{3}, x_{0} x_{1}^{4} x_{2}, x_{0}^{3} x_{1}^{3}\right\} \\
& =\operatorname{ICone}^{*}\left\{x_{0}^{4} x_{1}^{3} x_{2}, x_{0}^{3} x_{1}^{4} x_{2}^{3}\right\} .
\end{aligned}
$$

Definition $25\left(\partial_{p t}(i v)\right)$. Let iv be a three-dimensional inverted cotangent cone. The surface lattice points $\partial_{p t}(i v)$ of $i v$ is the set of all the $L^{* 3}$ lattice points on the surface of $i v$, i.e.,

$$
\begin{aligned}
\partial_{p t}(i v):=\{ & a\left(e_{3} / x_{i}\right)^{l}\left(e_{3} / x_{j}\right)^{m} \mid a \in \text { itop }(i v),\{i, j\} \subset\{0,1,2\}, 0 \geq l, m \in \mathbb{Z}, \\
& \left.a\left(e_{3} / x_{i}\right)^{l}\left(e_{3} / x_{j}\right)^{m} \notin \text { ICone }^{*}\left\{b e_{3}^{-2}\right\} \text { for } \forall b \in \text { itop }(i v)\right\} \subset L^{* 3} .
\end{aligned}
$$

Recall that $e_{3}=x_{0} x_{1} x_{2}$. For example, $e_{3} / x_{1}=x_{0} x_{2}$ and $e_{3}^{-2}=x_{0}^{-2} x_{1}^{-2} x_{2}^{-2}$.

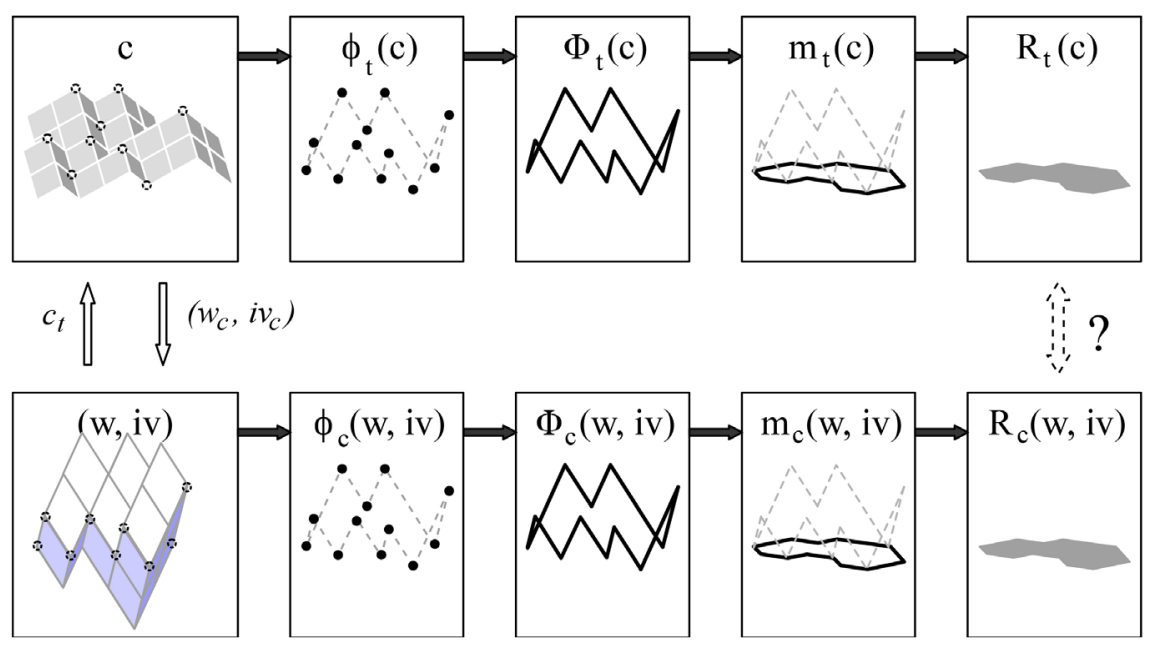

Figure 4. Schematic diagram showing the procedure for computing the contour of a region of $H_{2 D}$. The upper row shows the procedure for a region $R_{t}(c)$ specified by a tangent cone $c$. The lower row shows the procedure for a region $R_{c}(w, i v)$ specified by a contour pair $(w, i v)$. 
Definition 26 (Contour Pairs) Let $w$ be a three-dimensional cotangent cone. Let $i v$ be a three-dimensional inverted cotangent cone. A pair $(w, i v)$ of $w$ and $i v$ is called a three-dimensional contour pair.

Definition $27\left(\phi_{c}(w, i v)\right)$. Let $(w, i v)$ be a three-dimensional contour pair. The contour vertices $\phi_{c}(w, i v)$ with respect to $(w, i v)$ is defined by

$$
\phi_{c}(w, i v):=\partial_{p t}(w) \cap \partial_{p t}(i v) \subset L^{* 3} .
$$

That is, $\phi_{c}(w, i v)$ is the set of $L^{* 3}$ lattice points on the intersection of the surface of $w$ and the surface of $i v$.

Definition $28\left(\Phi_{c}(w, i v)\right)$. Let $(w, i v)$ be a three-dimensional contour pair. Let $\Phi_{c}(w, i v) \subset E^{3}$ be the polygonal line obtained by joining the adjacent $L^{* 3}$ lattice points of $\phi_{c}(w, i v)$ (Figure 3(c)). Since all the points of $\phi_{c}(w, i v)$ are on the surface of $w$ (or $i v)$, the points of $\phi_{c}(w, i v)$ are connected along the surface of $w$ (or $i v)$. Note that $\phi_{c}(w, i v)$ forms a closed polygonal line if top $(w)$ or itop $(i v)$ is finite (Figure $3(\mathrm{c}))$.

Definition $29\left(m_{c}(w, i v)\right)$. Let $(w, i v)$ be a three-dimensional contour pair. The one-dimensional surface mesh $m_{c}(w, i v)$ with respect to $(w, i v)$ is defined by

$$
m_{c}(w, i v):=\pi_{p t}\left(\Phi_{c}(w, i v)\right) \subset H_{2 D} .
$$

We also call $m_{c}(w, i v)$ the (one-dimensional) contour with respect to $(w, i v)$.

Remark Note that we have two types of one-dimensional surface meshes, i.e., the contour $m_{t}(c)$ with respect to a tangent cone $c$ and the contour $m_{c}(w, i v)$ with respect to a contour pair $(w, i v)$.

Definition $30\left(R_{c}(w, i v)\right)$. Let $(w, i v)$ be a three-dimensional contour pair. $R_{c}(w, i v) \subset H_{2 D}$ is the region enclosed by $m_{c}(w, i v)$ (Figure $3(\mathrm{c})$ ).

Definition $31\left(\left(w_{c}\left(c_{0}\right), i v_{c}\left(c_{0}\right)\right)\right)$. Let $c_{0}$ be a three-dimensional tangent cone. Suppose that $\operatorname{top}\left(c_{0}\right)$ is finite and $\partial_{t} \operatorname{top}\left(c_{0}\right) \subset L^{* 3}$. The three-dimensional contour pair $\left(w_{c}\left(c_{0}\right), i v_{c}\left(c_{0}\right)\right)$ associated with $c_{0}$ is a pair of the associated cotangent roof $w_{c}\left(c_{0}\right)$ and the inverted cotangent roof $i v_{c}\left(c_{0}\right)$ which is defined by

$$
i v_{c}\left(c_{0}\right):=\operatorname{IRoof}^{*} \phi_{t}\left(c_{0}\right) .
$$

Theorem 2 Let $c$ be a three-dimensional tangent cone. Suppose that top $(c)$ is finite and $\partial_{t}$ top $(c) \subset L^{* 3}$. Then,

$$
R_{t}(c)=R_{c}\left(w_{c}(c), i v_{c}(c)\right)
$$

Proof. Recall that

$$
\begin{aligned}
& \phi_{t}(c)=\partial_{p t}(c) \cap \partial_{p t}\left(w_{c}(c)\right), \\
& \phi_{c}\left(w_{c}(c), i v_{c}(c)\right)=\partial_{p t}\left(w_{c}(c)\right) \cap \partial_{p t}\left(i v_{c}(c)\right) .
\end{aligned}
$$

Note that $i v_{c}(c) \supset \phi_{t}(c)$, which implies $\partial_{p t}\left(i v_{c}(c)\right) \supset \phi_{t}(c)$ because the slope inclination of cotangent cones is steeper than that of tangent cones. On the other hand, $\partial_{p t}\left(w_{c}(c)\right) \supset \phi_{t}(c)$ by definition. Therefore, 
$\phi_{c}\left(w_{c}(c), i v_{c}(c)\right) \supset \phi_{t}(c)$. Suppose that $\phi_{c}\left(w_{c}(c), i v_{c}(c)\right) \neq \phi_{t}(c)$. Let $p$ be an $L^{* 3} \quad$ lattice point of $\phi_{c}\left(w_{c}(c), i v_{c}(c)\right)$ not included in $\phi_{t}(c)$. Then, $p \in \partial_{p t}\left(i v_{c}(c)\right)$ and $p \notin \partial_{p t}(c)$. That is, $p$ resides on the part of the surface of $i v_{c}(c)$ which is expanded by the "roof" operation. But the expanded part of $i v_{c}(c)$ is strictly contained in $w_{c}(c)$, i.e., $p \notin \partial_{p t}\left(w_{c}(c)\right)$, which is a contradiction. Therefore, $\phi_{t}(c)=\phi_{c}\left(w_{c}(c), i v_{c}(c)\right)$. In particular, $R_{t}(c)=R_{c}\left(w_{c}(c), i v_{c}(c)\right)$.

Example 10 In the case of Figure 3,

$$
\left\{\begin{aligned}
c_{0}=\text { Cone } & \left\{P_{a}, P_{b}, P_{c}, P_{d}, P_{e}\right\}, \\
w_{c}\left(c_{0}\right) & =\operatorname{Cone}^{*}\left\{K_{a}, K_{b}, K_{c}\right\}, \\
\operatorname{iv}_{c}\left(c_{0}\right) & =\operatorname{IRoof}^{*}\left\{P_{a}, P_{b}, P_{c}, P_{d}, P_{e}, P_{f}, P_{g}, P_{h}, P_{i}, P_{j}, P_{k}, P_{l}\right\} \\
& =\operatorname{IRoof}^{*}\left\{P_{f}, P_{h}, P_{i}, P_{k}\right\} \\
& =\operatorname{ICone}^{*}\left\{K_{d}, K_{e}\right\} .
\end{aligned}\right.
$$

In Theorem 2, we have computed the "contour" of $R_{t}(c)$ for a give tangent cone $c$ (Figure 5(a)). Now, we will compute the "contour" of $R_{c}(w, i v)$ for a given contour pair $(w, i v)$ (Figure $5(\mathrm{~b}))$.

Definition 32 Let $(w, i v)$ be a three-dimensional contour pair. The tangent cone $c_{t}(w, i v)$ associated with $(w, i v)$ is defined by

$$
c_{t}(w, i v):=\text { Cone } \phi_{c}(w, i v) \text {. }
$$

Note that $\operatorname{top}\left(c_{t}(w, i v)\right) \subset L^{* 3}$.

Theorem 3 Let $(w, i v)$ be a three-dimensional contour pair. Then,

$$
R_{c}(w, i v) \supset R_{t}\left(c_{t}(w, i v)\right) .
$$

Proof. Suppose that $\exists q \in H_{2 D}$ such that $q \in R_{t}\left(c_{t}(w, i v)\right)$ and $q \notin R_{c}(w, i v)$. Then, there exists an $L^{* 3}$ lattice point $p$ of $\phi_{t}\left(c_{t}(w, i v)\right)$ (i.e., points on the contour) such that $\pi_{p t}(p) \notin R_{c}(w, i v) \quad\left(\pi_{p t}(p)\right.$ is on the contour of $R_{t}\left(c_{t}(w, i v)\right)$.) It follows that $p$ is outside $i v$ because $p \in \partial_{p t}(w)$. However, the part of the surface of $w$ not included in $i v$ is strictly contained in $c$. Therefore, $p \notin \partial_{p t}\left(c_{t}(w, i v)\right)$, which is a contradiction.

Remark In general, $c_{t}(w, i v) \supset \phi_{c}(w, i v)$ does not imply $\partial_{p t}\left(c_{t}(w, i v)\right) \supset \phi_{c}(w, i v)$ because the slope inclination of tangent cones is gentler than that of cotangent cones. Therefore, there exists a contour pair $(w, i v)$ s.t.

$$
\begin{aligned}
\phi_{t}\left(c_{t}(w, i v)\right) & =\partial_{p t}\left(c_{t}(w, i v)\right) \cap \partial_{p t}\left(w_{c}\left(c_{t}(w, i v)\right)\right) \\
& =\partial_{p t}\left(c_{t}(w, i v)\right) \cap \partial_{p t}(w) \\
& \not \phi_{c}(w, i v) .
\end{aligned}
$$

In particular, $\phi_{t}\left(c_{t}(w, i v)\right) \neq \phi_{c}(w, i v)$, i.e., $R_{t}\left(c_{t}(w, i v)\right) \neq R_{c}(w, i v)$.

Starting with some definitions, we will consider the correspondence between the two types of regions of $H_{2 D}$, i.e., $R_{t}(c) s$ and $R_{c}(w, i v) \mathrm{s}$.

Definition 33 Sets of there-dimensional cones are defined by 


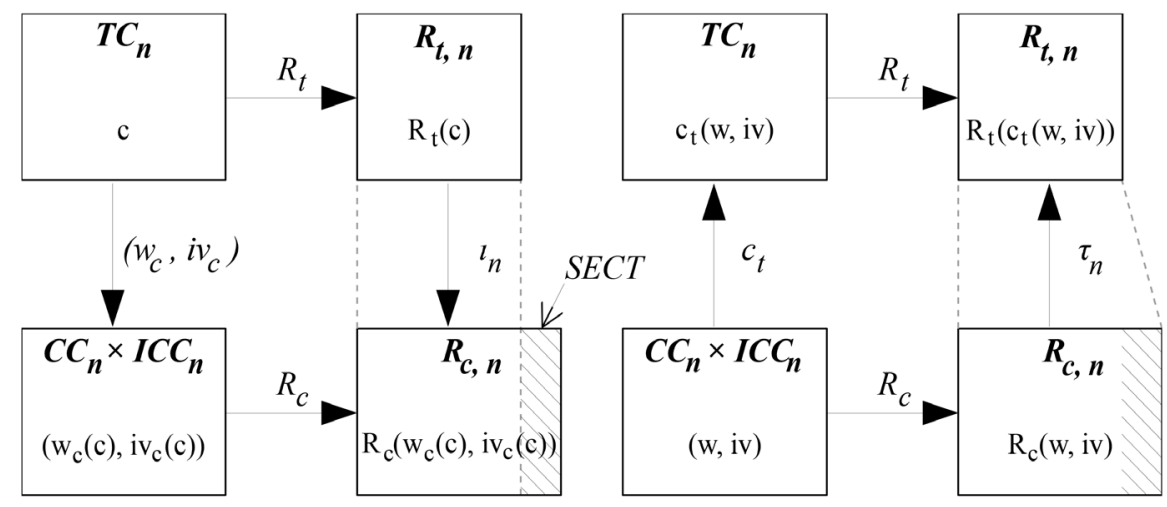

(a)

(b)

Figure 5. Maps between $R_{t, n}$ and $R_{c, n}$ and the relevant maps between sets of $n+1$ -dimensional cones ( $n=2$ or 3): (a) $t_{n}: R_{t, n} \hookrightarrow R_{c, n}$ and the relevant maps; (b) $\tau_{n}: R_{c, n} \rightarrow R_{t, n}$ and the relevant maps. The SECT denotes the set of all self-eclipsed closed trajectory complexes.

$$
\left\{\begin{array}{l}
T C_{2}:=\left\{\text { Cone } A \mid A \subset L^{* 3} \text { and } A \text { is finite }\right\}, \\
C C_{2}:=\left\{\text { Cone }^{*} A \mid A \subset L^{* 3} \text { and } A \text { is finite }\right\}, \\
I C C_{2}:=\left\{\text { ICone }^{*} A \mid A \subset L^{* 3} \text { and } A \text { is finite }\right\} .
\end{array}\right.
$$

Note that $\operatorname{top}(c) \subset L^{* 3}$ for $\forall c \in T C_{2}$.

Definition 34 ( $R_{t, 2}$ and $R_{c, 2}$ ) Sets of regions of $H_{2 D}$ are defined by

$$
\left\{\begin{array}{l}
R_{t, 2}:=\left\{R_{t}(c) \mid c \in T C_{2}\right\}, \\
R_{c, 2}:=\left\{R_{c}(w, i v) \mid(w, i v) \in C C_{2} \times I C C_{2}\right\} .
\end{array}\right.
$$

Definition 35 ( $l_{2}$ and $\tau_{2}$ ) Maps between regions of $H_{2 D}$ are defined by

$$
\left\{\begin{array}{l}
l_{2}: R_{t, 2} \hookrightarrow R_{c, 2}, R_{t}(c) \mapsto R_{c}\left(w_{c}(c), i v_{c}(c)\right), \\
\tau_{2}: R_{c, 2} \rightarrow R_{t, 2}, R_{c}(w, i v) \mapsto R_{t}\left(c_{t}(w, i v)\right) .
\end{array}\right.
$$

It is not difficult to show that the maps are well-defined. By Theorem 2, we have

$$
\iota_{2}\left(R_{t}(c)\right)=R_{t}(c)
$$

See Figure 5 for the correlation between the relevant maps.

Theorem $4 \quad l_{2}$ is not surjective.

Proof. Let us consider the case of Figure 6(a). We have

$$
\left\{\begin{array}{l}
w_{0}=\text { Cone }^{*}\left\{K_{a}\right\}, \\
i v_{0}=\operatorname{ICone}^{*}\left\{K_{b}\right\}, \\
\phi_{c}\left(w_{0}, i v_{0}\right)=\left\{P_{a}, P_{a b}, P_{b}, P_{c}, P_{c d}, P_{d}, P_{d e}, P_{e}, P_{f}, P_{f a}\right\}
\end{array}\right.
$$

where $K_{a}=x_{0}^{-1} x_{2}^{-1}, K_{b}=x_{0}^{2} x_{1}^{4} x_{2}^{2}, P_{a}=1, P_{a b}=x_{1} x_{2}, P_{b}=x_{1}^{2} x_{2}^{2}, \quad P_{c}=x_{0}^{-1} x_{1}^{2} x_{2}$, $P_{c d}=x_{1}^{3} x_{2}, P_{d}=x_{0} x_{1}^{4} x_{2}, P_{d e}=x_{0} x_{1}^{3}, P_{e}=x_{0} x_{1}^{2} x_{2}^{-1}, P_{f}=x_{0}^{2} x_{1}^{2}$, and $P_{f a}=x_{0} x_{1}$. Then, $R_{c}\left(w_{0}, i v_{0}\right)$ forms a flattened hexagon as shown in the figure. 


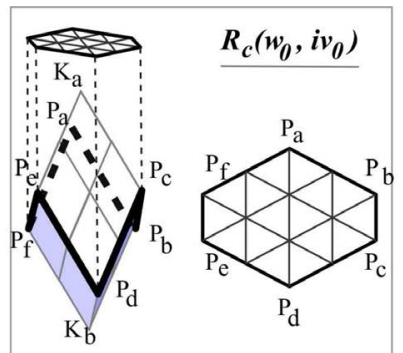

(a)

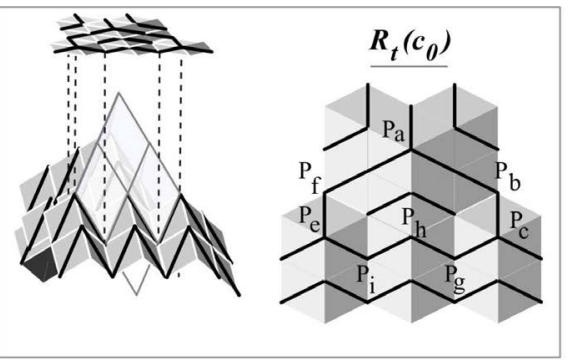

(b)

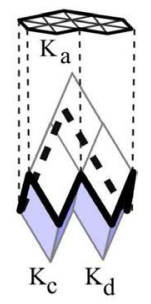

(c)

Figure 6. Self-eclipsed close trajectories: (a) Contour pair $\left(w_{0}, i v_{0}\right)$ and $R_{c}\left(w_{0}, i v_{0}\right)$; (b) The tangent cone $c_{0}:=c_{c}\left(w_{0}, i v_{0}\right)$ and the self-eclipsed region $R_{t}\left(c_{0}\right)$; (c) The contour pair $\left(w_{c}\left(c_{0}\right), i v_{c}\left(c_{0}\right)\right)$ associated with $c_{0}$.

On the other hand, the tangent cone $c_{t}\left(w_{0}, i v_{0}\right)$ and its associated cotangent roof is given by

$$
\left\{\begin{array}{l}
c_{t}\left(w_{0}, i v_{0}\right)=\text { Cone }\left\{P_{a}, P_{b}, P_{c}, P_{d}, P_{e}, P_{f}\right\}=\text { Cone }\left\{P_{a}, P_{c}, P_{e}\right\}, \\
w_{c}\left(c_{t}\left(w_{0}, i v_{0}\right)\right)=\text { Roof }^{*}\left\{P_{a}, P_{c}, P_{e}\right\}=\text { Cone }\left\{K_{a}\right\}, \\
\phi_{t}\left(c_{t}\left(w_{0}, i v_{0}\right)\right)=\left\{P_{a}, P_{a b}, P_{b}, P_{c}, P_{g}, P_{h}, P_{i}, P_{e}, P_{f}, P_{f a}\right\}
\end{array}\right.
$$

(Figure 6(b)). Then, the region $R_{t}\left(c_{t}\left(w_{0}, i v_{0}\right)\right)$ gets dented on the bottom, where $P_{g}=x_{1}^{3} x_{2}, P_{h}=x_{1}^{2}$, and $P_{i}=x_{0} x_{1}^{3}$. That is,

$$
\tau_{2}\left(R_{c}\left(w_{0}, i v_{0}\right)\right) \neq R_{c}\left(w_{0}, i v_{0}\right) \text {. }
$$

It follows immediately that $R_{c}\left(w_{0}, i v_{0}\right) \notin l_{2}\left(R_{t, 2}\right)$.

Corollary 2 (Self-eclipse of $R c(w, i v))$ There exists a contour pair $(w, i v)$ such that $\tau_{2}\left(R_{c}(w, i v)\right) \neq R_{c}(w, i v)$.

That is, not all closed polygonal lines defined by contour pairs correspond to a closed trajectory of triangles induced by a tangent cone. In other words, there exist global geometrical constraints on the contour of closed trajectories of triangles.

Definition 36 (Self-eclipsed closed trajectory complexes) $A$ contour pair $(w, i v)$ is called a self-eclipsed contour pair if $\tau_{2}\left(R_{c}(w, i v)\right) \neq R_{c}(w, i v)$. The complex of closed trajectories of triangles induced by a three-dimensional tangent cone $\mathrm{c}$ is called a self-eclipsed closed trajectory complex (abbreviated as SECT) if there exists a self-eclipsed contour pair $(w, i v)$ such that $c=c_{t}(w, i v)$.

Example 11 In the case of Figure 6(b),

$$
\begin{aligned}
& \tau_{2}\left(R_{c}\left(\text { Cone }^{*}\left\{K_{a}\right\}, \text { ICone }^{*}\left\{K_{b}\right\}\right)\right) \\
& =R_{t}\left(\text { Cone }\left\{P_{a}, P_{c}, P_{e}\right\}\right) \\
& =R_{c}\left(\text { Cone }^{*}\left\{K_{a}\right\}, \text { ICone }^{*}\left\{K_{c}, K_{d}\right\}\right) \\
& \neq R_{c}\left(\text { Cone }^{*}\left\{K_{a}\right\}, \text { ICone }^{*}\left\{K_{b}\right\}\right),
\end{aligned}
$$

where $K_{c}=x_{0}^{2} x_{1}^{3} x_{2}$ and $K_{d}=x_{0} x_{1}^{3} x_{2}^{2} \quad$ (Figure 6(c)). That is, the closed trajectory of Figure $6(\mathrm{~b})$ is a self-eclipsed closed trajectory complex. 
In the next section, we will consider geometrical constraints on the shape of closed trajectories of tetrahedrons. As an example, it will be shown that a garlic bulb-like structure appears as a result of the constraints, where a flattened dodecahedron gets dented on the bottom and has vertical linear grooves on the side (Figure 11).

\section{Mathematical Toy Model of Protein Molecules}

\subsection{Flows of Tetrahedrons}

Now let us consider the case of tetrahedrons. To define a flow of tetrahedrons, we use unit cubes in the four-dimensional Euclidean space $E^{4}$. By piling up unit cubes in the direction of $(-1,-1,-1,-1)$, we will obtain a flow of "slant" tetrahedrons as in the case of flows of triangles.

Definition 37 (Standard Lattice) The four-dimensional standard lattice $L^{4}$ is defined by

$$
L^{4}:=\left\{x_{0}^{l} x_{1}^{m} x_{2}^{n} x_{3}^{k} \mid l, m, n, k \in \mathbb{Z}\right\} \subset E^{4} .
$$

Let $p_{0}, p_{1}, p_{2}$, and $p_{3} \in L^{4}$. We denote the convex hull of four points $p_{0}$, $p_{1}, p_{2}$, and $p_{3}$ by $\left[p_{0}, p_{1}, p_{2}, p_{3}\right]$, i.e.,

$$
\left[p_{0}, p_{1}, p_{2}, p_{3}\right]:=\left\{p_{0}^{a} p_{1}^{b} p_{2}^{c} p_{3}^{d} \mid 0 \leq a, b, c, d \in \mathbb{R}, a+b+c+d=1\right\},
$$

We denote the group of all permutations of the four-element set $\{0,1,2,3\}$ by $\mathrm{Sym}^{4}$.

Definition 38 (Slant Tetrahedrons) Let $a \in L^{4}$ and $\rho \in S_{y m}{ }^{4}$. A slant tetrahedron $a\left[x_{\rho(0)} x_{\rho(1)} x_{\rho(2)}\right]$ is the tetrahedron defined by four points a, $a x_{\rho(0)}, \quad a x_{\rho(0)} x_{\rho(1)}$, and $a x_{\rho(0)} x_{\rho(1)} x_{\rho(2)}$, i.e.,

$$
a\left[x_{\rho(0)} x_{\rho(1)} x_{\rho(2)}\right]:=\left[a, a x_{\rho(0)}, a x_{\rho(0)} x_{\rho(1)}, a x_{\rho(0)} x_{\rho(1)} x_{\rho(2)}\right] .
$$

The line segment joining vertex $a$ and vertex $a x_{\rho(0)} x_{\rho(1)} x_{\rho(2)}$, i.e., the cube-diagonal, is called the diagonal edge of the slant tetrahedron. Then, the four upper faces of each unit cube are divided into six tetrahedrons along the diagonal edge as shown in Example 12. The set $S_{3}$ of all slant tetrahedrons is defined by:

$$
S_{3}:=\left\{a\left[x_{\rho(0)} x_{\rho(1)} x_{\rho(2)}\right] \mid a \in L^{4}, \rho \in \operatorname{Sym}^{4}\right\} .
$$

Example 12 Shown in Figure 7(a) is a four-dimensional unit cube at the origin $P_{1}$. The upper face $P_{1} P_{y} P_{y z} P_{z} P_{x} P_{x y} P_{x y z} P_{x x}$ of the cube is divided into six tetrahedrons along the cube-diagonal $P_{1} P_{x y z}$ :

$$
\left\{\begin{array}{l}
P_{1} P_{x} P_{x y} P_{x y z}=\left[x_{0} x_{1} x_{2}\right]=\left[1, x_{0}, x_{0} x_{1}, x_{0} x_{1} x_{2}\right], \\
P_{1} P_{y} P_{x y} P_{x y z}=\left[x_{1} x_{0} x_{2}\right]=\left[1, x_{1}, x_{1} x_{0}, x_{1} x_{0} x_{2}\right], \\
P_{1} P_{y} P_{y z} P_{x y z}=\left[x_{1} x_{2} x_{0}\right]=\left[1, x_{1}, x_{1} x_{2}, x_{1} x_{2} x_{0}\right], \\
P_{1} P_{z} P_{y z} P_{x y z}=\left[x_{2} x_{1} x_{0}\right]=\left[1, x_{2}, x_{2} x_{1}, x_{2} x_{1} x_{0}\right], \\
P_{1} P_{z} P_{x z} P_{x y z}=\left[x_{2} x_{0} x_{1}\right]=\left[1, x_{2}, x_{2} x_{0}, x_{2} x_{0} x_{1}\right], \\
P_{1} P_{x} P_{x z} P_{x y z}=\left[x_{0} x_{2} x_{1}\right]=\left[1, x_{0}, x_{0} x_{2}, x_{0} x_{2} x_{1}\right] .
\end{array}\right.
$$




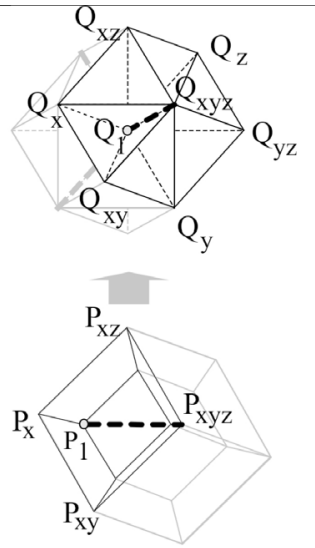

(a)

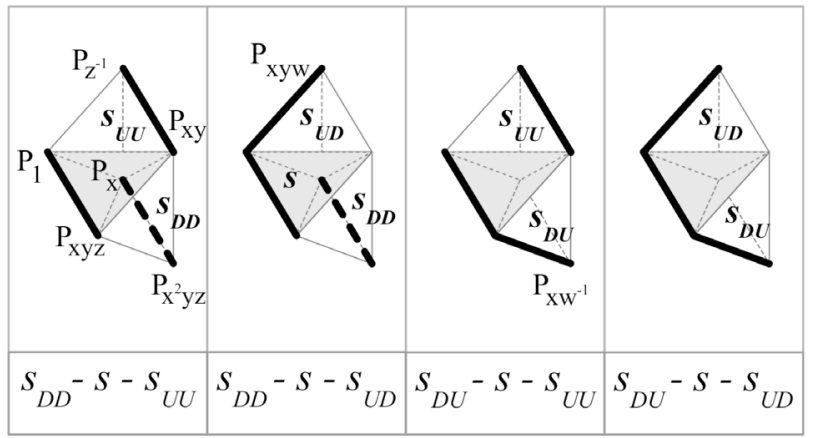

(b)

Figure 7. Slant tetrahedrons: (a) A four-dimensional unit cube shown in the Schlegel diagram (below) and its projection image on hyperplane $H_{3 D}$ (above), where $P_{x^{l} y^{m} z^{n} w^{k}}=x_{0}^{l} x_{1}^{m} x_{2}^{n} x_{3}^{k} \in L^{4} \quad$ and $\quad Q_{x^{l} y^{m} z^{n} w^{k}}=\pi_{p t}\left(x_{0}^{l} x_{1}^{m} x_{2}^{n} x_{3}^{k}\right) \in H_{3 D} ; \quad$ (b) All the local trajectories at $s=P_{1} P_{x} P_{x y} P_{x y z} \in B_{3}$. The diagonal edges of tetrahedrons are drawn with thick lines.

Definition 39 (Gradient of Slant Tetrahedrons) Let $s=a\left[x_{\rho(0)} x_{\rho(1)} x_{\rho(2)}\right] \in S_{4}$. The gradient Ds of $s$ is defined by

$$
D s:=x_{\rho(0)} x_{\rho(1)} x_{\rho(2)} .
$$

By abuse of notation, we denote vector $(l, m, n, k)$ by monomial $x_{0}^{l} x_{1}^{m} x_{2}^{n} x_{3}^{k}$ in the definition.

Example 13 In the case of Example 12,

$$
D\left(P_{1} P_{x} P_{x y} P_{x y z}\right)=D\left[x_{0} x_{1} x_{2}\right]=x_{0} x_{1} x_{2}
$$

Flows of slant tetrahedrons along the diagonal edges are defined as follows.

Definition 40 (Local Trajectories of Slant Tetrahedrons) Let $s \in S_{3}$. A local trajectory of slant tetrahedrons at $s$ is a set of three consecutive slant tetrahedrons, consisting of $s$ and two adjacent slant tetrahedrons which do not include the diagonal edge of $s$. By patching "consistent" local trajectories together, we obtain a flow of slant tetrahedrons.

Let $s=a\left[x_{\rho(0)} x_{\rho(1)} x_{\rho(2)}\right] \in S_{3}$. Then, the local trajectory of slant tetrahedrons at $s$ is either

$$
\left\{s_{D D}, s, s_{U U}\right\} \text { or }\left\{s_{D D}, s, s_{U D}\right\} \text { or }\left\{s_{D U}, s, s_{U U}\right\} \text { or }\left\{s_{D U}, s, s_{U D}\right\},
$$

where

$$
\left\{\begin{array}{l}
s_{D D}:=a x_{\rho(0)}\left[x_{\rho(1)} x_{\rho(2)} x_{\rho(0)}\right] \in S_{3}, \\
s_{D U}:=a x_{\rho(0)} x_{\rho(3)}^{-1}\left[x_{\rho(3)} x_{\rho(1)} x_{\rho(2)}\right] \in S_{3}, \\
s_{U D}:=a\left[x_{\rho(0)} x_{\rho(1)} x_{\rho(3)}\right] \in S_{3}, \\
s_{U U}:=a x_{\rho(2)}^{-1}\left[x_{\rho(2)} x_{\rho(0)} x_{\rho(1)}\right] \in S_{3} .
\end{array}\right.
$$


Example 14 In the case of Example 13, the four local trajectories at $P_{1} P_{x} P_{x y} P_{x y z} \quad\left(=\left[x_{0} x_{1} x_{2}\right]\right)$ are shown in Figure $7(\mathrm{~b})$.

A flow of "flat" tetrahedrons is defined on the hyperplane $H_{3 D}$ using the projection $\pi_{p t}$ of $E^{4}$ onto $H_{3 D}$, where

$$
\left\{\begin{array}{l}
H_{3 D}:=\left\{x_{0}^{l} x_{1}^{m} x_{2}^{n} x_{3}^{k} \mid l, m, n, k \in \mathbb{R}, l+m+n+k=0\right\} \subset E^{4}, \\
\pi_{p t}: E^{4} \rightarrow H_{3 D}, \pi_{p t}\left(x_{0}^{l} x_{1}^{m} x_{2}^{n} x_{3}^{k}\right) \\
:=x_{0}^{(3 x-y-z-w) / 4} x_{1}^{(-x+3 y-z-w) / 4} x_{2}^{(-x-y+3 z-w) / 4} x_{3}^{(-x-y-z+3 w) / 4} .
\end{array}\right.
$$

Definition 41 (Flat Tetrahedrons) Let $s=a\left[x_{\rho(0)} x_{\rho(1)} x_{\rho(2)}\right] \in S_{3}$. The projection $\pi_{H}$ of $s$ on $H_{3 D}$ is defined by

$$
\pi_{H}(s):=\left[\pi_{p t}(a), \pi_{p t}\left(a x_{\rho(0)}\right), \pi_{p t}\left(a x_{\rho(0)} x_{\rho(1)}\right), \pi_{p t}\left(a x_{\rho(0)} x_{\rho(1)} x_{\rho(2)}\right)\right] \subset H_{3 D} .
$$

$\pi_{H}(s)$ is called a flat tetrahedron. The line segment joining $\pi_{p t}(a)$ and $\pi_{p t}\left(a x_{\rho(0)} x_{\rho(1)} x_{\rho(2)}\right)$ is called the diagonal edge of the flat tetrahedron. The set $B_{3}$ of all flat tetrahedrons on $H_{3 D}$ is defined by:

$$
B_{3}:=\left\{\pi_{H}(s) \mid s \in S_{3}\right\} .
$$

Example 15 In the case of Example 12 (Figure 7(a)), the projection image $Q_{1} Q_{x} Q_{x y} Q_{y} Q_{z} Q_{x z} Q_{x y z} Q_{y z}$ of the upper face $P_{1} P_{x} P_{x y} P_{y} P_{z} P_{x z} P_{x y z} P_{y z}$ by $\pi_{L}$ is divided into six flat tetrahedrons:

$$
\left\{\begin{array}{l}
Q_{1} Q_{x} Q_{x y} Q_{x y z}=\pi_{H}\left(\left[x_{0} x_{1} x_{2}\right]\right), \\
Q_{1} Q_{y} Q_{x y} Q_{x y z}=\pi_{H}\left(\left[x_{1} x_{0} x_{2}\right]\right), \\
Q_{1} Q_{y} Q_{y z} Q_{x y z}=\pi_{H}\left(\left[x_{1} x_{2} x_{0}\right]\right), \\
Q_{1} Q_{z} Q_{y z} Q_{x y z}=\pi_{H}\left(\left[x_{2} x_{1} x_{0}\right]\right), \\
Q_{1} Q_{z} Q_{x z} Q_{x y z}=\pi_{H}\left(\left[x_{2} x_{0} x_{1}\right]\right), \\
Q_{1} Q_{x} Q_{x z} Q_{x y z}=\pi_{H}\left(\left[x_{0} x_{2} x_{1}\right]\right) .
\end{array}\right.
$$

Remark. Note that each tetrahedron has two long edges and four short edges, where the diagonal edge correspond to a short edge. Flows of tetrahedrons go along the diagonal edge at each tetrahedron.

By projecting slant tetrahedrons onto $H_{3 D}$, we obtain a three-dimensional flow of flat tetrahedrons on $H_{3 D}$.

Definition 42 (Local Trajectories of Flat Tetrahedrons) The local trajectory of flat tetrahedrons at $t \in B_{3}$ is a projection image of a local trajectory of slant tetrahedrons at $s \in S_{3}$ by $\pi_{H}$, where $\pi_{H}(s)=t$. The local trajectory at $s$ is given by either

$$
\begin{aligned}
& \left\{\pi_{H}\left(s_{D D}\right), \pi_{H}(s), \pi_{H}\left(s_{U U}\right)\right\} \text { or }\left\{\pi_{H}\left(s_{D D}\right), \pi_{H}(s), \pi_{H}\left(s_{U D}\right)\right\} \\
& \text { or }\left\{\pi_{H}\left(s_{D U}\right), \pi_{H}(s), \pi_{H}\left(s_{U U}\right)\right\} \text { or }\left\{\pi_{H}\left(s_{D U}\right), \pi_{H}(s), \pi_{H}\left(s_{U D}\right)\right\} .
\end{aligned}
$$

Note that $\pi_{H}\left(s_{D D}\right)=\pi_{H}\left(s_{D U}\right)$ and $\pi_{H}\left(s_{U D}\right)=\pi_{H}\left(s_{U U}\right)$. By patching "consistent" local trajectories together, we obtain a flow of flat tetrahedrons as shown in Figure 8. 


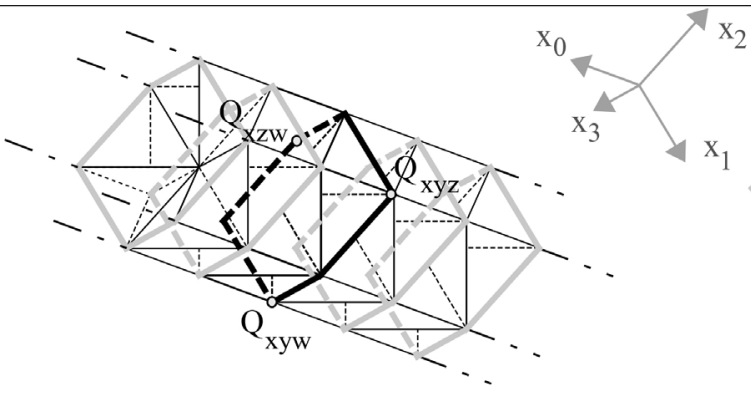

(a)

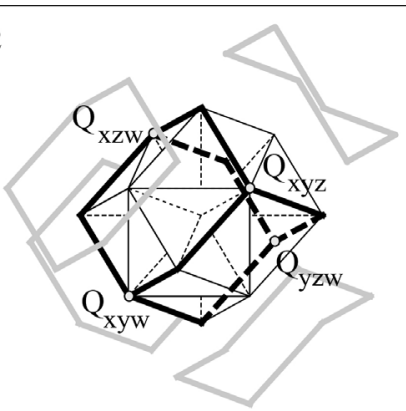

(b)

Figure 8. Flows of tetrahedrons: (a) Closed trajectories of flat tetrahedrons induced by $c_{0}=$ Cone $\left\{Q_{x y z}, Q_{x z w}, Q_{x y w}\right\}$, where $Q_{x y z}=x_{0} x_{1} x_{2}, Q_{x z w}=x_{0} x_{2} x_{3}$, and $Q_{x y w}=x_{0} x_{1} x_{3}$. Thick polygonal lines (black and grey) indicate the diagonal edges of flat tetrahedrons, where the black line indicate the polygonal line passing through $Q_{x y z}, Q_{x z w}$, and $Q_{x y w}$. Note that there exist infinitely many closed trajectories of length six and length twelve; (b) Closed trajectories of flat tetrahedrons induced by $c_{1}=$ Cone $\left\{Q_{x y z}, Q_{x z w}, Q_{x y w}, Q_{y z w}\right\}$, where $Q_{y z w}=x_{1} x_{2} x_{3}$. Thick polygonal lines (black and grey) indicate the diagonal edges of flat tetrahedrons. Black lines indicate the polygonal lines passing through $Q_{x y z}, Q_{x z w}, Q_{x y w}$, or $Q_{y z w}$. Grey polygonal lines correspond to closed trajectories of length six and length twelve.

\subsection{Vector Fields of Tetrahedrons}

The tangent space on the space $B_{3}$ of flat tetrahedrons is defined in the same way as the tangent space $T B_{2}$ on $B_{2}$.

Definition 43 (Tangent Space) The tangent space $T B_{3}$ on $B_{3}$ is defined by

$$
\left\{\begin{array}{l}
T B_{3}:=B_{3} \times\left\{x_{1} x_{2} x_{3}, x_{0} x_{2} x_{3}, x_{0} x_{1} x_{3}, x_{0} x_{1} x_{2}\right\}, \\
\pi: T B_{3} \rightarrow B_{3}, \quad \pi\left(\left(t, x_{i} x_{j} x_{k}\right)\right):=t .
\end{array}\right.
$$

Let $t \in B_{3}$. The tangent space at $t$ is denoted by $T B_{3}[t]$. Note that there exists a one-to-one correspondence

$$
T B_{3}[t] \sim\left\{x_{1} x_{2} x_{3}, x_{0} x_{2} x_{3}, x_{0} x_{1} x_{3}, x_{0} x_{1} x_{2}\right\} .
$$

Tangent cones are also defined similarly for $A \subset L^{4}$.

Definition 44 (Tangent Cones) Let $A \subset L^{4}$. The four-dimensional tangent cone Cone $A$ is defined by

$$
\text { Cone } A:=\left\{a x_{0}^{l} x_{1}^{m} x_{2}^{n} x_{3}^{k} \mid a \in A, 0 \leq l, m, n, k \in \mathbb{Z}\right\} \subset L^{4} .
$$

The set of all the top vertices of a cone $c$ is denoted by $\operatorname{top}(c)$.

Definition $45 \partial_{t} \operatorname{top}(c)$. Let $\mathrm{c}$ be a four-dimensional tangent cone. The peaks on the boundary of $\mathrm{c}$ is defined by

$$
\begin{aligned}
\partial_{t} \operatorname{top}(c):= & \{p \in \operatorname{top}(c) \mid \exists i \in\{0,1,2,3\} \text { s.t. } \\
& \left.p\left(x_{i}\right)^{N} \notin \operatorname{Cone}\{\operatorname{top}(c) \backslash\{p\}\} \text { for } \forall N \in \mathbb{N}\right\} .
\end{aligned}
$$

Example 16 In the case of Figure 8, 


$$
\begin{aligned}
& \partial_{t} \operatorname{top}\left(c_{0}\right)=\left\{Q_{x y z}, Q_{x z w}, Q_{x y w}\right\}, \\
& \partial_{t} \operatorname{top}\left(c_{1}\right)=\left\{Q_{x y z}, Q_{x z w}, Q_{x y w}, Q_{y z w}\right\} .
\end{aligned}
$$

Definition $46\left(\partial_{p t}(c)\right)$. Let $c$ be a four-dimensional tangent cone. The surface lattice points $\partial_{p t}(c)$ of $c$ is the set of all the $L^{4}$ lattice points included in the 3 -faces of $c$, i.e.,

$$
\begin{aligned}
\partial_{p t}(c):= & \left\{a x_{i}^{l} x_{j}^{m} x_{k}^{n} \mid a \in \operatorname{top}(c),\{i, j, k\} \subset\{0,1,2,3\}, 0 \leq l, m, k \in \mathbb{Z},\right. \\
& \left.a x_{i}^{l} x_{j}^{m} x_{k}^{n} \notin \text { Cone }\left\{b x_{0} x_{1} x_{2} x_{3}\right\} \text { for } \forall b \in \operatorname{top}(c)\right\} \subset L^{4} .
\end{aligned}
$$

Example 17 The surface lattice points of the four-dimensional tangent cone Cone $\{1\}$ are given by

$$
\partial_{p t}(\text { Cone }\{1\})=U_{l=0} \cup U_{m=0} \cup U_{n=0} \cup U_{k=0} \subset L^{4},
$$

where

$$
\left\{\begin{array}{l}
U_{l=0}:=\left\{x_{1}^{m} x_{2}^{n} x_{3}^{k} \mid 0 \leq m, n, k \in \mathbb{Z}\right\}, \\
U_{m=0}:=\left\{x_{0}^{l} x_{2}^{n} x_{3}^{k} \mid 0 \leq l, n, k \in \mathbb{Z}\right\}, \\
U_{n=0}:=\left\{x_{0}^{l} x_{1}^{m} x_{3}^{k} \mid 0 \leq l, m, k \in \mathbb{Z}\right\}, \\
U_{k=0}:=\left\{x_{0}^{l} x_{1}^{m} x_{2}^{n} \mid 0 \leq l, m, n \in \mathbb{Z}\right\} .
\end{array}\right.
$$

That is, $\partial_{p t}($ Cone $\{1\})$ is the union of the four 3 -faces of Cone $\{1\}$.

Definition $47\left(d_{S} c\right)$ Let $c$ be a four-dimensional tangent cone. Then, $d_{S} c$ is the set of all the slant tetrahedrons included in the 3-faces of $c$, i.e.

$$
d_{S} c:=\left\{a\left[x_{\rho(0)} x_{\rho(1)} x_{\rho(2)}\right] \in S_{3} \mid \text { all the vertices are included in } \partial_{p t}(c)\right\} .
$$

Definition 48 (Vector Fields) Let $c$ be a four-dimensional tangent cone. The vector field $V_{c}$ induced by $c$ on $B_{3}$ is defined by

$$
V_{c}(t):=D s\left(t \in B_{3}\right),
$$

where $s \in d_{S} c \cap \pi_{H}^{-1}(t)$ (Note that $s$ is uniquely determined for each $t \in B_{3}$ ). The flow of tetrahedrons determined by $V_{c}$ is called the flow of tetrahedrons induced by $c$.

Example 18 In the case of Figure 7(a),

$$
V_{c}\left(Q_{1} Q_{x} Q_{x y} Q_{x y z}\right)=D\left(P_{1} P_{x} P_{x y} P_{x y z}\right)=x_{0} x_{1} x_{2},
$$

where $c=$ Cone $\{1\}, Q_{1} Q_{x} Q_{x y} Q_{x y z}=\pi_{H}\left(P_{1} P_{x} P_{x y} P_{x y z}\right)$, and $P_{1} P_{x} P_{x y} P_{x y z}=\left[x_{0} x_{1} x_{2}\right]$.

Unlike the case of flows of triangles, infinitely many closed trajectories are induced by a tangent cone.

Example 19 Shown in Figure 8(a) is the closed trajectories of the flow induced by Cone $\left\{x_{0} x_{1} x_{2}, x_{0} x_{2} x_{3}, x_{0} x_{1} x_{3}\right\}$. Two types of closed trajectories, one is length 6 and the other is length 12, are alternately stacked infinitely.

Example 20 By putting another top vertex $x_{1} x_{2} x_{3}$ on the tangent cone of Figure 8(a), we obtain a decomposition of a rhombic dodecahedron into four closed trajectories of tetrahedrons (Figure $8(\mathrm{~b})$ ). Then, each triplet of the four top vertices of Cone $\left\{x_{0} x_{1} x_{2}, x_{0} x_{2} x_{3}, x_{0} x_{1} x_{3}, x_{1} x_{2} x_{3}\right\}$ induces infinitely many closed 
trajectories outside the rhombic dodecahedron (grey polygonal lines).

\subsection{Shape of Closed Trajectories of Tetrahedrons}

To compute the surface (2-faces) of the region of $H_{3 D}$ swept by a set of closed trajectories of flat tetrahedrons, we will consider another type of cones whose ridge lines are given by the "slopes" of slant tetrahedrons, i.e., $(0,1,1,1)$, $(1,0,1,1),(1,1,0,1)$, and $(1,1,1,0)$.

Definition 49 (Conjugate Lattice) The four-dimensional conjugate lattice $L^{* 4}$ is defined by

$$
L^{* 4}:=\left\{x_{0}^{m+n+k} x_{1}^{l+n+k} x_{2}^{l+m+k} x_{3}^{l+m+n} \mid l, m, n, k \in \mathbb{Z}\right\} \subset L^{4} .
$$

Definition 50 (Cotangent Cones) Let $A \subset L^{* 4}$. The four-dimensional cotangent cone $\operatorname{Cone}^{*} A$ is defined by

$$
\begin{aligned}
\text { Cone }^{*} A:= & \left\{a\left(x_{1} x_{2} x_{3}\right)^{l}\left(x_{0} x_{2} x_{3}\right)^{m}\left(x_{0} x_{1} x_{3}\right)^{n}\left(x_{0} x_{1} x_{2}\right)^{k} \mid a \in A,\right. \\
& 0 \leq l, m, n, k \in \mathbb{Z}\} \subset L^{* 4} .
\end{aligned}
$$

We denote the top vertices of a cotangent cone $c$ by $\operatorname{top}(c)$.

Definition 51 (Cotangent Roofs) Let $A \subset L^{* 4}$. The four-dimensional cotangent roof $\operatorname{Roof}^{*} A$ is defined by

$$
\begin{aligned}
\text { Roof }^{*} A:= & \left\{p \in L^{* 4} \mid \exists N \in \mathbb{Z} \text { s.t. } N>0 \text { and } p\left(x_{1} x_{2} x_{3}\right)^{N},\right. \\
& \left.p\left(x_{0} x_{2} x_{3}\right)^{N}, p\left(x_{0} x_{1} x_{3}\right)^{N}, p\left(x_{0} x_{1} x_{2}\right)^{N} \in \text { Cone }^{*} A\right\} .
\end{aligned}
$$

Example 21 In the case of Figure 8,

$$
\begin{aligned}
\operatorname{Roof}^{*} \partial_{t} \operatorname{top}\left(c_{0}\right) & =\operatorname{Roof}^{*}\left\{x_{0} x_{1} x_{2}, x_{0} x_{2} x_{3}, x_{0} x_{1} x_{3}\right\} \\
& =\operatorname{Cone}^{*}\left\{x_{0} x_{1} x_{2}, x_{0} x_{2} x_{3}, x_{0} x_{1} x_{3}\right\}, \\
\operatorname{Roff}^{*} \partial_{t} \operatorname{top}\left(c_{1}\right)= & \operatorname{Rof}^{*}\left\{x_{0} x_{1} x_{2}, x_{0} x_{2} x_{3}, x_{0} x_{1} x_{3}, x_{1} x_{2} x_{3}\right\} \\
= & \operatorname{Cone}^{*}\{1\} .
\end{aligned}
$$

Definition $52\left(\partial_{p t}(w)\right)$. Let $\mathrm{w}$ be a four-dimensional cotangent cone. The surface lattice points $\partial_{p t}(w)$ of $w$ is the set of all the $L^{* 4}$ lattice points included in the 3 -faces of $w$, i.e.,

$$
\begin{aligned}
\partial_{p t}(w):= & \left\{a\left(e_{4} / x_{i}\right)^{l}\left(e_{4} / x_{j}\right)^{m}\left(e_{4} / x_{k}\right)^{n} \mid a \in \operatorname{top}(w),\{i, j, k\} \subset\{0,1,2,3\},\right. \\
& 0 \leq l, m, n \in \mathbb{Z}, a\left(e_{4} / x_{i}\right)^{l}\left(e_{4} / x_{j}\right)^{m}\left(e_{4} / x_{k}\right)^{n} \notin \operatorname{Cone}^{*}\left\{b e_{4}^{3}\right\} \\
& \text { for } \forall b \in \operatorname{top}(w)\} \subset L^{* 4},
\end{aligned}
$$

where $e_{4}:=x_{0} x_{1} x_{2} x_{3} \quad$ (For example, $e_{4} / x_{1}=x_{0} x_{2} x_{3}$ and $e_{4}^{3}=x_{0}^{3} x_{1}^{3} x_{2}^{3} x_{3}^{3}$ ).

Example 22 The surface lattice points of the four-dimensional cotangent cone Cone $^{*}\{1\}$ are given by

$$
\partial_{p t}\left(\text { Cone }^{*}\{1\}\right)=V_{l=0} \cup V_{m=0} \cup V_{n=0} \cup V_{k=0} \subset L^{* 4},
$$

where 


$$
\left\{\begin{array}{l}
V_{l=0}:=\left\{\left(x_{0} x_{2} x_{3}\right)^{m}\left(x_{0} x_{1} x_{3}\right)^{n}\left(x_{0} x_{1} x_{2}\right)^{k} \mid 0 \leq m, n, k \in \mathbb{Z}\right\}, \\
V_{m=0}:=\left\{\left(x_{1} x_{2} x_{3}\right)^{l}\left(x_{0} x_{1} x_{3}\right)^{n}\left(x_{0} x_{1} x_{2}\right)^{k} \mid 0 \leq l, n, k \in \mathbb{Z}\right\}, \\
V_{n=0}:=\left\{\left(x_{1} x_{2} x_{3}\right)^{l}\left(x_{0} x_{2} x_{3}\right)^{m}\left(x_{0} x_{1} x_{2}\right)^{k} \mid 0 \leq l, m, k \in \mathbb{Z}\right\}, \\
V_{k=0}:=\left\{\left(x_{1} x_{2} x_{3}\right)^{l}\left(x_{0} x_{2} x_{3}\right)^{m}\left(x_{0} x_{1} x_{3}\right)^{n} \mid 0 \leq l, m, n \in \mathbb{Z}\right\} .
\end{array}\right.
$$

As in the case of slant triangles, all the slant tetrahedrons of the flow induced by a tangent cone are divided into three groups by a cotangent cone: 1) inside the cotangent cone, 2) outside the cotangent cone, and3) on the surface (3-faces) of the cotangent cone (Figure 9). Unlike the case of triangles, multiple types of slant tetrahedrons are on the surface (3-faces) of the cotangent cone (tetrahedrons B, C, D, E, F in Figure 9). Among them, only two types of tetrahedrons (B and F) flow through the surface (2-faces).

Definition $53\left(w_{c}\left(c_{0}\right)\right)$. Let $c_{0}$ be a four-dimensional tangent cone. Suppose that $\partial_{t}$ top $\left(c_{0}\right) \subset L^{* 4}$. The four-dimensional cotangent roof $w_{c}\left(c_{0}\right)$ associated with $c_{0}$ is defined by

$$
w_{c}\left(c_{0}\right):=\operatorname{Roof}^{*} \partial_{t} \operatorname{top}\left(c_{0}\right) .
$$

Definition $54\left(\phi_{t}\left(c_{0}\right)\right)$. Let $c_{0}$ be a four-dimensional tangent cone. Suppose that $\partial_{t} \operatorname{top}\left(c_{0}\right) \subset L^{* 4}$. The surface vertices $\phi_{t}\left(c_{0}\right)$ with respect to $c_{0}$ is defined by

$$
\phi_{t}\left(c_{0}\right):=\partial_{p t}\left(c_{0}\right) \cap \partial_{p t}\left(w_{c}\left(c_{0}\right)\right) \subset L^{* 4} .
$$

That is, $\phi_{t}\left(c_{0}\right)$ is the set of $L^{* 4}$ lattice points on the intersection of the 3-faces of $c_{0}$ and the 3-faces of $w_{c}\left(c_{0}\right)$.

Definition $55\left(\Phi_{t}\left(c_{0}\right)\right)$. Let $c_{0}$ be a four-dimensional tangent cone. Suppose that $\partial_{t}$ top $\left(c_{0}\right) \subset L^{* 4}$. Let $\Phi_{t}\left(c_{0}\right) \subset E^{4}$ be the set of the polygonal lines obtained by joining the adjacent $L^{* 4}$ lattice points of surface vertices $\phi_{t}\left(c_{0}\right)$. Since all the points of $\phi_{t}\left(c_{0}\right)$ are included in the 3-faces of $w_{c}\left(c_{0}\right)$, the points of $\phi_{t}\left(c_{0}\right)$ are connected along the 3 -faces of the associated cotangent roof.

Definition $56\left(m_{t}\left(c_{0}\right)\right)$. Let $c_{0}$ be a four-dimensional tangent cone. The two-dimensional surface mesh $m_{t}\left(c_{0}\right)$ with respect to $c_{0}$ is defined by

$$
m_{t}\left(c_{0}\right):=\pi_{p t}\left(\Phi_{t}\left(c_{0}\right)\right) \subset H_{3 D} .
$$

We also call $m_{t}\left(c_{0}\right)$ (two-dimensional) surface vein with respect to $c_{0}$.

Definition 57 Let $c_{0}$ be a four-dimensional tangent cone. Set

$$
\left\{\begin{array}{l}
I N_{t}\left(c_{0}\right):=\left\{s \in d_{S} c_{0} \mid s \text { is contained inside } w_{c}\left(c_{0}\right)\right\}, \\
O U T_{t}\left(c_{0}\right):=\left\{s \in d_{S} c_{0} \mid s \text { is contained outside } w_{c}\left(c_{0}\right)\right\}, \\
B D_{t}\left(c_{0}\right):=\left\{s \in d_{S} c_{0} \mid s \text { intersects with the } 3 \text {-faces of } w_{c}\left(c_{0}\right)\right\} .
\end{array}\right.
$$

Definition $58\left(R_{t}\left(c_{0}\right)\right)$. Let $c_{0}$ be a four-dimensional tangent cone. We define the region $R_{t}\left(c_{0}\right)$ of $H_{3 D}$ by 


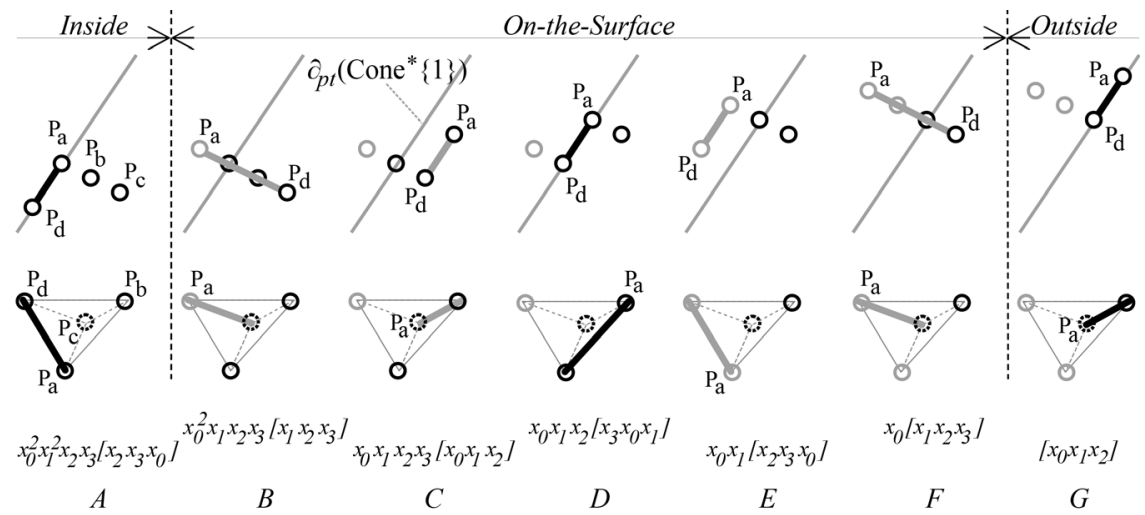

Figure 9. Positional relationship of slant triangles of $\pi_{H}^{-1}\left(\left[x_{0} x_{1} x_{2}\right]\right)$ and the 3 -face $\left\{\left(x_{0} x_{2} x_{3}\right)^{m}\left(x_{0} x_{1} x_{3}\right)^{n}\left(x_{0} x_{1} x_{2}\right)^{k} \mid 0 \leq m, n, k \in \mathbb{Z}\right\}$ of the cotangent cone Cone $\{1\}$. Slant triangles shown are A: $x_{0}^{2} x_{1}^{2} x_{2} x_{3}\left[x_{2} x_{3} x_{0}\right], \mathrm{B}: x_{0}^{2} x_{1} x_{2} x_{3}\left[x_{1} x_{2} x_{3}\right], \mathrm{C}: x_{0} x_{1} x_{2} x_{3}\left[x_{0} x_{1} x_{2}\right], \mathrm{D}:$ $x_{0} x_{1} x_{2}\left[x_{3} x_{0} x_{1}\right], \mathrm{E}: x_{0} x_{1}\left[x_{2} x_{3} x_{0}\right], \mathrm{F}: x_{0}\left[x_{1} x_{2} x_{3}\right]$, and G: $\left[x_{0} x_{1} x_{2}\right]$. Shown above is a schematic diagram of the relationship between the four vertices of a slant tetrahedron and the 3-face of $\operatorname{Cone}^{*}\{1\}$. In the figure, the diagonal edges of slant tetrahedrons are drawn with thick line, where the diagonal edges included in the 3 -face are colored black and the others are colored grey. Tetrahedron A is included in $\operatorname{Cone}^{*}\{1\}$. Tetrahedrons B, C, D, E, and $\mathrm{F}$ intersect the 3 -face of $\operatorname{Cone}^{*}\{1\}$. Tetrahedron G is located outside Cone ${ }^{*}\{1\}$. Note that the diagonal edges of tetrahedrons B and F cross the 3-face of Cone $\{1\}$.

$$
R_{t}\left(c_{0}\right):=\bigcup_{s \in I N_{t}\left(c_{0}\right)} \pi_{H}(s) \subset H_{3 D} .
$$

In the case of flows of triangles, $R_{t}(c)$ corresponds to all the closed trajectories of $V_{c}$ if $\operatorname{top}(c)$ is finite and $\partial_{t} \operatorname{top}(c) \subset L^{* 3}$ (Theorem 1). In the case of flows of tetrahedrons, we have the following result.

Theorem 5 There exist a four-dimensional tangent cone $c$ such that $R_{t}(c)$ does not contain all the closed trajectories of $V_{c}$.

Proof. For example, set $c_{0}=$ Cone $^{*}\left\{x_{0} x_{1} x_{2}, x_{0} x_{2} x_{3}, x_{0} x_{1} x_{3}\right\}$ (Figure 8(a)). Then, $V_{c_{0}}$ induces infinitely many closed trajectories of tetrahedrons. However, we can not construct a cotangent cone which covers all the closed trajectories because $w_{c}\left(c_{0}\right)=c_{0}$ (Note that more than three vertices are required to construct a "roof" on a tangent cone.) In particular, $R_{t}\left(c_{0}\right)$ does not contain all the closed trajectories of $V_{c_{0}}$.

As in the case of flows of triangles, we can compute $R_{t}(c)$ as the intersection of $w_{c}(c)$ and $c$ if $\partial_{t}$ top $(c) \subset L^{* 4}$.

Example 23 In the case of Figure 8(b) (or Figure10(a)),

$$
\left\{\begin{array}{l}
c_{1}=\text { Cone }\left\{x_{0} x_{1} x_{2}, x_{0} x_{2} x_{3}, x_{0} x_{1} x_{3}, x_{1} x_{2} x_{3}\right\}, \\
w_{c}\left(c_{1}\right)=\text { Roof }^{*}\left\{x_{0} x_{1} x_{2}, x_{0} x_{2} x_{3}, x_{0} x_{1} x_{3}, x_{1} x_{2} x_{3}\right\}=\text { Cone }^{*}\{1\}, \\
\phi_{t}\left(c_{1}\right)=\left\{P_{y z w}, P_{x z w}, P_{x y w}, P_{x y z}, P_{x^{2} y^{2} z w}, P_{x^{2} y z^{2} w}, P_{x^{2} y z w^{2}}, P_{x y^{2} z^{2} w^{2}}, P_{x y^{2} z w^{2}}, P_{x y z^{2} w^{2}}\right\},
\end{array}\right.
$$

where $P_{x^{l} y^{m} z^{n} w^{k}}=x_{0}^{l} x_{1}^{m} x_{2}^{n} x_{3}^{k} \in L^{4}$. Then, 


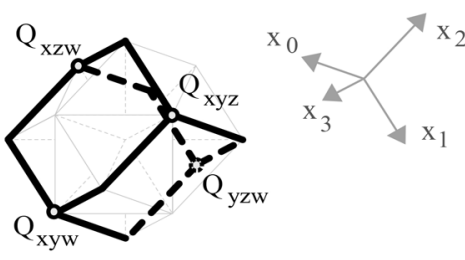

(a)

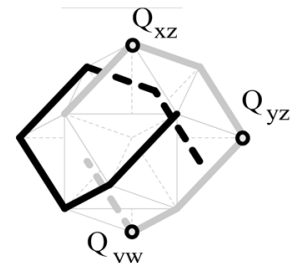

(b)

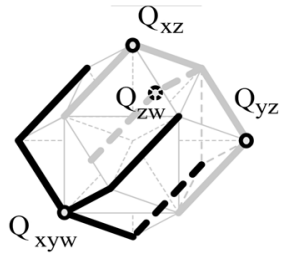

(c)

Figure 10. Rhombic dodecahedrons: (a) Four closed trajectories of the flow induced by $c_{1}=$ Cone $\left\{x_{0} x_{1} x_{2}, x_{0} x_{2} x_{3}, x_{0} x_{1} x_{3}, x_{1} x_{2} x_{3}\right\} \quad$ (See also Figure 8 (b)); (b) The closed trajectory of length 24 induced by $c_{2}=$ Cone $\left\{x_{0} x_{2}, x_{1} x_{2}, x_{1} x_{3}\right\}$; (c) The closed trajectory of length 24 induced by $c_{3}=$ Cone $\left\{x_{0} x_{2}, x_{1} x_{2}, x_{2} x_{3}, x_{0} x_{1} x_{3}\right\}$. Thick polygonal lines (black and grey) indicate the diagonal edges of flat tetrahedrons, where the black lines indicate the polygonal lines included in $w_{c}\left(c_{i}\right) \quad(i=1,2,3)$.

$R_{t}\left(c_{1}\right)$ is the rhombic dodecahedron surrounded by the black thick lines, which consists of four closed trajectories of length six, i.e., consists of 24 tetrahedrons.

All the 24 tetrahedrons of $R_{t}\left(c_{1}\right)$ are type D of Figure 9:

1) $Q_{1}$ is inside $w_{c}\left(c_{1}\right)$,

2) $Q_{x^{2} y z w}, Q_{x y^{2} z w}, Q_{x y z^{2} w}$ and $Q_{x y z w^{2}}$ are outside $w_{c}\left(c_{1}\right)$,

3) all the diagonal edges of the tetrahedrons $\subset \partial_{p t}\left(w_{c}\left(c_{1}\right)\right)$,

where $Q_{x^{l} y^{m} z^{n} w^{k}}=\pi_{p t}\left(x_{0}^{l} x_{1}^{m} x_{2}^{n} x_{3}^{k}\right) \in H_{3 D}$. Other closed trajectories of the vector field are outside $w_{c}\left(c_{1}\right)$.

In the case of flows of tetrahedrons, we should also consider the case of $\partial_{t}$ top $(c) \not \subset L^{* 4}$.

Example 24 Putting more unit cubes on the tangent cone $c_{1}$ of Figure10(a), we obtain rhombic dodecaherons consisting of a closed trajectory of length 24 (Figure 10(b) and Figure 10(c)). However, we can not compute the shape of the rhombic dodecaherons using cotangent roofs. Cotangent roofs are not defined because

$$
\partial_{t} t o p\left(c_{i}\right) \not \subset L^{* 4} \quad(i=2,3) .
$$

Remark In the three-dimensional case, $\partial_{t}$ top $(c) \not \subset L^{* 3}$ implies the existence of loopholes on the contour, i.e., the existence of the triangles of type D (Figure 2 (b)). On the other hand, in the four-dimensional case, $\partial_{t}$ top $(c) \not \subset L^{* 4}$ implies not only the existence of loopholes but also dents and bulges on the surface. That is, the tetrahedrons of type $\mathrm{B}$ and $\mathrm{F}$ correspond to loopholes, the tetrahedrons of type $\mathrm{C}$ to dents, and the tetrahedrons of type $\mathrm{E}$ to bulges (Figure 9).

To define a four-dimensional cotangent roof $\operatorname{Roof}^{*} A$ for any $A \subset L^{4}$, we consider a set of the "closest $L^{* 4}$ lattice points to a" for each $a \in A$.

Definition 59 (STAND) Let $a \in L^{4}$. The stand of a is defined by

$$
\operatorname{stand}(a):= \begin{cases}\{a\} & \text { if } \operatorname{deg}(a) \equiv 0 \bmod 3, \\ \left\{a x_{i} \mid 0 \leq i \leq 3\right\} & \text { if } \operatorname{deg}(a) \equiv 2 \bmod 3, \\ \left\{a x_{i} x_{j} \mid 0 \leq i, j \leq 3\right\} & \text { if } \operatorname{deg}(a) \equiv 1 \bmod 3,\end{cases}
$$


where $\operatorname{deg}\left(x_{0}^{l} x_{1}^{m} x_{2}^{n} x_{3}^{k}\right):=l+m+n+k$. Note that $\operatorname{stand}(a) \subset L^{* 4}$.

Let $A \subset L^{4}$. The stand of $A$ is defined by

$$
\operatorname{STAND}(A):=\bigcup_{a \in A} \text { stand }(a) \subset L^{* 4} .
$$

Definition 60 (Extended Cotangent Roofs) Let $A \subset L^{4}$ such that $A \not \subset L^{* 4}$. The four-dimensional extended cotangent roof Eroof ${ }^{*} A$ is defined by

$$
\operatorname{Eroof}^{*} A:=\operatorname{Roof}^{*} \operatorname{STAND}(A) \text {. }
$$

Definition 61 (Extended $\left.w_{c}\left(c_{0}\right)\right)$ Let $c_{0}$ be a four-dimensional tangent cone. The four-dimensional (extended) cotangent roof $w_{c}\left(c_{0}\right)$ associated with $c_{0}$ is defined by

$$
w_{c}\left(c_{0}\right):=\operatorname{Eroof}^{*} \partial_{t} \text { top }\left(c_{0}\right)
$$

Note that

$$
\operatorname{Eroof}^{*} \partial_{t} \operatorname{top}\left(c_{0}\right)=\operatorname{Roof}^{*} \partial_{t} \operatorname{top}\left(c_{0}\right)
$$

if $\partial_{t}$ top $\left(c_{0}\right) \subset L^{* 4}$.

Definition 62 (Extended $\left.\phi_{t}\left(c_{0}\right)\right)$ Let $c_{0}$ be a four-dimensional tangent cone. The (extended) surface vertices $\phi_{t}\left(c_{0}\right)$ with respect to $c_{0}$ is defined by

$$
\phi_{t}\left(c_{0}\right):=\partial_{p t}\left(c_{0}\right) \cap \partial_{p t}\left(w_{c}\left(c_{0}\right)\right) \subset L^{* 4} .
$$

Example 25 In the case of Figure 10(b),

$$
\left\{\begin{array}{l}
c_{2}=\text { Cone }\left\{x_{0} x_{2}, x_{1} x_{2}, x_{1} x_{3}\right\}, \\
w_{c}\left(c_{2}\right)=\text { Eroof }\left\{x_{0} x_{2}, x_{1} x_{2}, x_{1} x_{3}\right\}=\text { Cone }^{*}\{1\}, \\
\phi_{t}\left(c_{2}\right)=\left\{P_{x z w}, P_{x^{2} y z w^{2}}, P_{x y w}, P_{x^{2} y^{2} z w}, P_{x y z}, P_{x y z^{2} w^{2}}, P_{y z w}\right\} .
\end{array}\right.
$$

Then, $R_{t}\left(c_{2}\right)$ is the rhombic dodecahedron surrounded by the black and grey thick lines, which consists of a closed trajectories of length 24 .

Note that $x_{0} x_{2}, x_{1} x_{2}$, and $x_{1} x_{3}$ are outside Cone ${ }^{*}\{1\}$. The rhombic dodecahedron consists of not only type D but also type E tetrahedrons of Figure 9. In the figure, the tetrahedron with the grey diagonal edge are type E. The tetrahedrons with the black diagonal edge are type D.

Example 26 In the case of Figure 10(c),

$$
\left\{\begin{array}{l}
c_{3}=\text { Cone }\left\{x_{0} x_{2}, x_{1} x_{2}, x_{2} x_{3}, x_{0} x_{1} x_{3}\right\}, \\
w_{c}\left(c_{3}\right)=\text { Eroof }^{*}\left\{x_{0} x_{2}, x_{1} x_{2}, x_{2} x_{3}, x_{0} x_{1} x_{3}\right\}=\text { Cone }^{*}\{1\}, \\
\phi_{t}\left(c_{3}\right)=\left\{P_{x z w}, P_{x^{2} y z w^{2}}, P_{x y w}, P_{x^{2} y^{2} z w}, P_{x y z}, P_{x y^{2} z w^{2}}, P_{y z w}\right\} .
\end{array}\right.
$$

Then, $R_{t}\left(c_{3}\right)$ is the rhombic dodecahedron surrounded by the black and grey thick lines, which consists of a closed trajectories of length 24 . In the figure, the tetrahedrons with the grey diagonal edge are type $\mathrm{E}$. The tetrahedrons with the black diagonal edge are type $\mathrm{D}$.

\subsection{Constraints on the Shape of Protein Molecules}

In this paper, we consider the shape of complexes of closed trajectories of 
tetrahedrons as a simplified geometrical model of protein molecules. As in the case of flows of triangles, we will specify the shape of regions of $H_{3 D}$ using a pair of cotangent cones.

Definition 63 (Inverted Cotangent Cones) Let $A \subset L^{* 4}$. The four-dimensional inverted cotangent cone ICone $A$ is defined by

$$
\begin{aligned}
\text { ICone }^{*} A:= & \left\{a\left(x_{1} x_{2} x_{3}\right)^{l}\left(x_{0} x_{2} x_{3}\right)^{m}\left(x_{0} x_{1} x_{3}\right)^{n}\left(x_{0} x_{1} x_{2}\right)^{k} \mid a \in A,\right. \\
& 0 \geq l, m, n, k \in \mathbb{Z}\} \subset L^{*_{4} .}
\end{aligned}
$$

We denote the top vertices of an inverted cotangent cone iv by itop $(i v)$.

Definition 64 (Inverted Cotangent Roofs) Let $A \subset L^{* 4}$. The four-dimensional inverted cotangent roof $\operatorname{IRoof~}^{*} A$ is defined by

$$
\begin{aligned}
\text { IRoof }^{*} A:= & \left\{p \in L^{* 4} \mid \exists N \in \mathbb{Z} \text { s.t. } N<0 \text { and } p\left(x_{1} x_{2} x_{3}\right)^{N},\right. \\
& \left.p\left(x_{0} x_{2} x_{3}\right)^{N}, p\left(x_{0} x_{1} x_{3}\right)^{N}, p\left(x_{0} x_{1} x_{2}\right)^{N} \in \text { ICone }^{*} A\right\} .
\end{aligned}
$$

Let $A \subset L^{4}$. The four-dimensional extended inverted cotangent roof EIroof $^{*} A$ is defined by

$$
\text { EIroof }^{*} A:=\operatorname{IRoof}^{*} \operatorname{STAND}(A) \subset L^{* 4} .
$$

Example 27 In the case of Figure 10(a),

$$
\begin{aligned}
& \text { IRoof }^{*} \partial_{t} \operatorname{top}\left(c_{1}\right) \\
& =\text { IRoof }^{*}\left\{x_{0} x_{1} x_{2}, x_{0} x_{2} x_{3}, x_{0} x_{1} x_{3}, x_{1} x_{2} x_{3}\right\} \\
& =\text { ICone }^{*}\left\{x_{0}^{3} x_{1}^{3} x_{2}^{3} x_{3}^{3}\right\} .
\end{aligned}
$$

Definition $65\left(\partial_{p t}(i v)\right)$. Let iv be a four-dimensional inverted cotangent cone. The surface lattice points $\partial_{p t}(i v)$ of $i v$ is the set of all the $L^{* 4}$ lattice points included in the 3 -faces of $i v$, i.e.,

$$
\begin{aligned}
\partial_{p t}(i v):= & \left\{a\left(e_{4} / x_{i}\right)^{l}\left(e_{4} / x_{j}\right)^{m}\left(e_{4} / x_{k}\right)^{n} \mid a \in \text { itop }(i v),\{i, j, k\} \subset\{0,1,2,3\},\right. \\
& 0 \geq l, m, n \in \mathbb{Z}, a\left(e_{4} / x_{i}\right)^{l}\left(e_{4} / x_{j}\right)^{m}\left(e_{4} / x_{k}\right)^{n} \notin I \text { Cone }^{*}\left\{b e_{4}^{-3}\right\} \\
& \text { for } \forall b \in \text { itop }(i v)\} \subset L^{* 4} .
\end{aligned}
$$

Recall that $e_{4}=x_{0} x_{1} x_{2} x_{3}$. For example, $e_{4} / x_{2}=x_{0} x_{1} x_{3}$ and $e_{4}^{-3}=x_{0}^{-3} x_{1}^{-3} x_{2}^{-3} x_{3}^{-3}$.

Definition 66 (Surface Pairs) Let $\mathrm{w}$ be a three-dimensional cotangent cone. Let iv be a three-dimensional inverted cotangent cone. A pair $(w, i v)$ of $w$ and $i v$ is called a four-dimensional surface pair.

Definition $67\left(\phi_{c}(w, i v)\right)$. Let $(w, i v)$ be a four-dimensional surface pair. The surface vertices $\phi_{c}(w, i v)$ with respect to $(w, i v)$ is defined by

$$
\phi_{c}(w, i v):=\partial_{p t}(w) \cap \partial_{p t}(i v) \subset L^{* 4} .
$$

That is, $\phi_{c}(w, i v)$ is the set of $L^{* 4}$ lattice points on the intersection of the 3 -faces of $w$ and the 3 -faces of $i v$.

Definition $68\left(\Phi_{c}(w, i v)\right)$. Let $(w, i v)$ be a four-dimensional surface pair. 
Let $\Phi_{c}(w, i v) \subset E^{3}$ be the set of the polygonal lines obtained by joining the adjacent $L^{* 4}$ lattice points of $\phi_{c}(w, i v)$. That is, $\Phi_{(w, i v)}$ is the set of all the diagonal edges (of slat tetrahedrons) whose end points are included in $\phi_{c}(w, i v)$. Since all the points of $\phi_{c}(w, i v)$ are on the 3-faces of $w$ (or $\left.i v\right)$, the points of $\phi_{c}(w, i v)$ are connected along the 3-face of $w$ (or $\left.i v\right)$.

Definition $69\left(m_{c}(w, i v)\right)$. Let $(w, i v)$ be a four-dimensional surface pair. The two-dimensional surface mesh $m_{c}(w, i v)$ with respect to $(w, i v)$ is defined by

$$
m_{c}(w, i v):=\pi_{p t}\left(\Phi_{c}(w, i v)\right) \subset H_{3 D} .
$$

We also call $m_{c}(w, i v)$ (two-dimensional) surface vein with respect to $(w, i v)$.

Definition $70\left(\left(w_{c}\left(c_{0}\right), i v_{c}\left(c_{0}\right)\right)\right)$. Let $c_{0}$ be a four-dimensional tangent cone. The four-dimensional surface pair $\left(w_{c}\left(c_{0}\right), i v_{c}\left(c_{0}\right)\right)$ associated with $c_{0}$ is a pair of the (extended) cotangent roof $w_{c}\left(c_{0}\right)$ associated with $c_{0}$ and the (extended) inverted cotangent roof $i v_{c}\left(c_{0}\right)$ associated with $c_{0}$ which is defined by

$$
i v_{c}\left(c_{0}\right):=\operatorname{EIroof}^{*} \partial_{t} \operatorname{top}\left(c_{0}\right) .
$$

Definition $71\left(R_{c}(w, i v)\right)$. Let $(w, i v)$ be a four-dimensional surface pair. The region $R_{c}(w, i v)$ of $H_{3 D}$ is the region covered by the union of all the closed trajectories of flat tetrahedrons surrounded by $m_{c}(w, i v)$.

Example 28 In the case of Figure 10(a),

$$
\left\{\begin{aligned}
w_{c}\left(c_{1}\right)=\text { Roof }^{*}\left\{x_{0} x_{1} x_{2}, x_{0} x_{2} x_{3}, x_{0} x_{1} x_{3}, x_{1} x_{2} x_{3}\right\}=\operatorname{Cone}^{*}\{1\}, \\
i v_{c}\left(c_{1}\right)=\operatorname{IRoof}^{*}\left\{x_{0} x_{1} x_{2}, x_{0} x_{2} x_{3}, x_{0} x_{1} x_{3}, x_{1} x_{2} x_{3}\right\}=\operatorname{ICone}^{*}\left\{x_{0}^{3} x_{1}^{3} x_{2}^{3} x_{3}^{3}\right\}, \\
\phi_{c}\left(w_{c}\left(c_{1}\right), i v_{c}\left(c_{1}\right)\right)=\left\{P_{y z w}, P_{x z w}, P_{x y w}, P_{x y z}, P_{x^{2} y^{2} z w^{2}}, P_{x^{2} y z^{2} w},\right. \\
\left.P_{x^{2} y z w^{2}}, P_{x y^{2} z^{2} w^{2}}, P_{x y y^{2} w^{2}}, P_{x y z^{2} w^{2}}\right\} .
\end{aligned}\right.
$$

Then, the surface vein $m_{c}\left(w_{c}\left(c_{1}\right), i v_{c}\left(c_{1}\right)\right)$ corresponds to the set of the diagonal edges colored black. $R_{c}\left(w_{c}\left(c_{1}\right), i v_{c}\left(c_{1}\right)\right)$ is the rhombic dodecahedron consisting of four closed trajectories of length four.

Example 29 In the case of Figure 10(b),

$$
\left\{\begin{array}{l}
w_{c}\left(c_{2}\right)=\text { Eroof }^{*}\left\{x_{0} x_{2}, x_{1} x_{2}, x_{1} x_{3}\right\}=\operatorname{Cone}^{*}\{1\}, \\
i_{c}\left(c_{2}\right)=\text { EIroof }^{*}\left\{x_{0} x_{2}, x_{1} x_{2}, x_{1} x_{3}\right\}=\operatorname{ICone}^{*}\left\{x_{0}^{3} x_{1}^{3} x_{2}^{3} x_{3}^{3}\right\}, \\
\phi_{c}\left(w_{c}\left(c_{2}\right), i v_{c}\left(c_{2}\right)\right)=\left\{P_{x z w}, P_{x^{2} y z w^{2}}, P_{x y w}, P_{x^{2} y^{2} z w}, P_{x y z}, P_{x y z^{2} w^{2}}, P_{y z w}\right\} .
\end{array}\right.
$$

Then, the surface vein $m_{c}\left(w_{c}\left(c_{2}\right), i v_{c}\left(c_{2}\right)\right)$ corresponds to the set of the diagonal edges colored black. Unlike the case of flows of triangles, some diagonal edges (colored grey) are not included in $m_{c}\left(w_{c}\left(c_{2}\right), i v_{c}\left(c_{2}\right)\right)$.

$R_{c}\left(w_{c}\left(c_{2}\right), i v_{c}\left(c_{2}\right)\right)$ is the rhombic dodecahedron consisting of a closed trajectories of length 24 . In particular, 


$$
R_{c}\left(w_{c}\left(c_{2}\right), i v_{c}\left(c_{2}\right)\right)=R_{c}\left(w_{c}\left(c_{1}\right), i v_{c}\left(c_{1}\right)\right) .
$$

Example 30 In the case of Figure 10(c),

$$
\left\{\begin{array}{l}
w_{c}\left(c_{3}\right)=\text { Eroof }^{*}\left\{x_{0} x_{2}, x_{1} x_{2}, x_{2} x_{3}, x_{0} x_{1} x_{3}\right\}=\operatorname{Cone}^{*}\{1\}, \\
\operatorname{iv}_{c}\left(c_{3}\right)=\text { Eroof }^{*}\left\{x_{0} x_{2}, x_{1} x_{2}, x_{2} x_{3}, x_{0} x_{1} x_{3}\right\}=\operatorname{ICone}^{*}\left\{x_{0}^{3} x_{1}^{3} x_{2}^{3} x_{3}^{3}\right\}, \\
\phi_{c}\left(w_{c}\left(c_{3}\right), i v_{c}\left(c_{3}\right)\right)=\left\{P_{x z w}, P_{x^{2} y z w^{2}}, P_{x y w}, P_{x^{2} y^{2} z w}, P_{x y z}, P_{x y^{2} z w^{2}}, P_{y z w}\right\} .
\end{array}\right.
$$

Then, the surface vein $m_{c}\left(w_{c}\left(c_{3}\right), i v_{c}\left(c_{3}\right)\right)$ corresponds to the set of the diagonal edges colored black. The diagonal edges colored grey are not included in $m_{c}\left(w_{c}\left(c_{3}\right), i v_{c}\left(c_{3}\right)\right) . R_{c}\left(w_{c}\left(c_{3}\right), i v_{c}\left(c_{3}\right)\right)$ is also the rhombic dodecahedron consisting of another closed trajectories of length 24. In particular,

$$
R_{c}\left(w_{c}\left(c_{3}\right), i v_{c}\left(c_{3}\right)\right)=R_{c}\left(w_{c}\left(c_{1}\right), i v_{c}\left(c_{1}\right)\right) \text {. }
$$

Example 31 In the case of Figure 8(a),

$$
\left\{\begin{array}{l}
w_{c}\left(c_{0}\right)=\text { Eroof }^{*}\left\{x_{0} x_{1} x_{2}, x_{0} x_{2} x_{3}, x_{0} x_{1} x_{3}\right\}=\text { Cone }^{*}\left\{x_{0} x_{1} x_{2}, x_{0} x_{2} x_{3}, x_{0} x_{1} x_{3}\right\}, \\
i_{c}\left(c_{0}\right)=\text { EIroof }^{*}\left\{x_{0} x_{1} x_{2}, x_{0} x_{2} x_{3}, x_{0} x_{1} x_{3}\right\}=\text { ICone }^{*}\left\{x_{0} x_{1} x_{2}, x_{0} x_{2} x_{3}, x_{0} x_{1} x_{3}\right\}, \\
\phi_{c}\left(w_{c}\left(c_{0}\right), i v_{c}\left(c_{0}\right)\right)=\left\{P_{x y z}, P_{x^{2} y z^{2} w}, P_{x z w}, P_{x^{2} y z w^{2}}, P_{x y w}, P_{x^{2} y^{2} z w}\right\} .
\end{array}\right.
$$

Then, the surface vein $m_{c}\left(w_{c}\left(c_{0}\right), i v_{c}\left(c_{0}\right)\right)$ is empty.

In the above examples, a tangent cone $c$ is given first. Then, we compute the surface vein $m_{c}\left(w_{c}(c), i v_{c}(c)\right)$ for $\left(w_{c}(c), i v_{c}(c)\right)$ (Figure 5(a)). Now, let us consider the case where a surface pair $(w, i v)$ is given first (Figure $5(\mathrm{~b})$ ).

Definition 72 Let $(w, i v)$ be a four-dimensional surface pair. The tangent cone $c_{t}(w, i v)$ associated with $(w, i v)$ is defined by

$$
c_{t}(w, i v):=\text { Cone }_{c}(w, i v) .
$$

Starting with some definitions, we will consider the vector field induced by the tangent cone $c_{t}(w, i v)$ associated with the given surface pair $(w, i v)$.

Definition 73 Sets of four-dimensional cones are defined by

$$
\begin{aligned}
& T C_{3}:=\left\{\text { Cone } A \mid A \subset L^{* 4} \text { and } A \text { is finite }\right\}, \\
& C C_{3}:=\left\{\text { Cone }^{*} A \mid A \subset L^{* 4} \text { and } A \text { is finite }\right\}, \\
& I C C_{3}:=\left\{\text { ICone }^{*} A \mid A \subset L^{* 4} \text { and } A \text { is finite }\right\} .
\end{aligned}
$$

Definition $74\left(R_{t, 3}\right.$ and $\left.R_{c, 3}\right)$ Sets of regions on $H_{2 D}$ are defined by

$$
\left\{\begin{array}{l}
R_{t, 3}:=\left\{R_{t}(c) \mid c \in T C_{3}\right\}, \\
R_{c, 3}:=\left\{R_{c}(w, i v) \mid(w, i v) \in C C_{3} \times I C C_{3}\right\} .
\end{array}\right.
$$

Definition 75 ( $l_{3}$ and $\tau_{3}$ ) Maps between regions on $H_{3 D}$ are defined by

$$
\left\{\begin{array}{l}
l_{3}: R_{t, 3} \hookrightarrow R_{c, 3}, \quad R_{t}(c) \mapsto R_{c}\left(w_{c}(c), i v_{c}(c)\right), \\
\tau_{3}: R_{c, 3} \rightarrow R_{t, 3}, \quad R_{c}(w, i v) \mapsto R_{t}\left(c_{t}(w, i v)\right) .
\end{array}\right.
$$

Theorem $6 l_{3}$ is not surjective. 
Proof. Let us consider the case of Figure 11(a), where

$$
\left\{\begin{array}{l}
\left(w_{s}, i v_{s}\right)=\left(\operatorname{Cone}^{*}\left\{P_{e}\right\}, \operatorname{ICone}^{*}\left\{P_{f}\right\}\right) \\
R_{c}\left(w_{s}, i v_{s}\right)=\text { a flattened dodecahedron. }
\end{array}\right.
$$

On the other hand, shown in Figure 11(b) are the closed trajectories of $\tau_{3}\left(R_{c}\left(w_{s}, i v_{s}\right)\right)=R_{t}\left(c_{t}\left(w_{s}, i v_{s}\right)\right)$, where

$$
\left\{\begin{array}{l}
c_{t}\left(w_{s}, i v_{s}\right)=\text { Cone }\left\{P_{a}, P_{b}, P_{c}, P_{d}\right\}, \\
w_{c}\left(c_{t}\left(w_{s}, i v_{s}\right)\right)=\text { Cone }^{*}\left\{P_{e}\right\}, \\
R_{t}\left(c_{t}\left(w_{s}, i v_{s}\right)\right)=\text { a garlic bulb-like structure. }
\end{array}\right.
$$

Note that $Q_{p}$ dose not appear because $P_{p}$ is buried beneath the surface of $c_{t}\left(w_{s}, i v_{s}\right)$ due to the difference of slope inclination between tangent cones and cotangent cones. The flattened dodecahedron of (a) gets dented on the bottom and has vertical linear grooves on the side. That is, we obtain a garlic bulb-like structure as a result of the constraints.

Therefore,

$$
\tau_{3}\left(R_{c}\left(w_{s}, i v_{s}\right)\right) \neq R_{c}\left(w_{s}, i v_{s}\right) .
$$

It follows immediately that $R_{c}\left(w_{s}, i v_{s}\right) \notin l_{3}\left(R_{t, 3}\right)$.

Corollary 3 (Self-eclipse of $\left.R_{c}(w, i v)\right)$ There exists a four-dimensional surface pair $(w, i v)$ such that $\tau_{3}\left(R_{c}(w, i v)\right) \neq R_{c}(w, i v)$.

That is, there exist global geometrical constraints on the shape of complexes of closed trajectories of tetrahedrons.

Definition 76 (Self-eclipsed protein molecules (Toy model)) A surface pair $(w, i v)$ is called a self-eclipsed surface pair if $\tau_{3}\left(R_{c}(w, i v)\right) \neq R_{c}(w, i v)$. The complex of closed trajectories induced by a tangent cone $c$ is called a self-eclipsed protein molecule if there exists a self-eclipsed surface pair $(w, i v)$ such that $c=c_{t}(w, i v)$.

Example 32 In the case of the garlic bulb-like structure obtained in the proof of Theorem 6 (Figure 11(a) and Figure 11(b)),

$$
\begin{aligned}
& \tau_{3}\left(R_{c}\left(\text { Cone }^{*}\left\{P_{e}\right\}, \text { ICone }^{*}\left\{P_{f}\right\}\right)\right) \\
& =R_{t}\left(\text { Cone }\left\{P_{a}, P_{b}, P_{c}, P_{d}\right\}\right) \\
& =R_{c}\left(\text { Cone }^{*}\left\{P_{e}\right\}, \text { ICone }^{*}\left\{P_{g}, P_{h}, P_{i}\right\}\right) \\
& \neq R_{c}\left(\text { Cone }^{*}\left\{P_{e}\right\}, \text { ICone } e^{*}\left\{P_{f}\right\}\right) \text {. }
\end{aligned}
$$

Example 33 Shown in Figure 11(c) is a complex of closed trajectories of $R_{t}\left(c_{1}\right)$, where

$$
\left\{\begin{array}{l}
c_{1}=\text { Cone }\left\{P_{b}, P_{c}, P_{d}, P_{j}, P_{k}, P_{l}, P_{m}, P_{n}, P_{o}\right\}, \\
w_{c}\left(c_{1}\right)=\text { Cone }^{*}\left\{P_{e}\right\}, \\
R_{t}\left(c_{1}\right)=\text { a garlic bulb-like structure. }
\end{array}\right.
$$



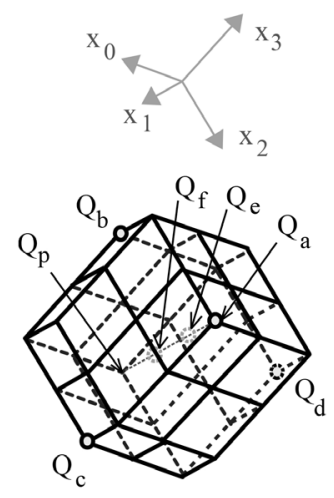

(a)

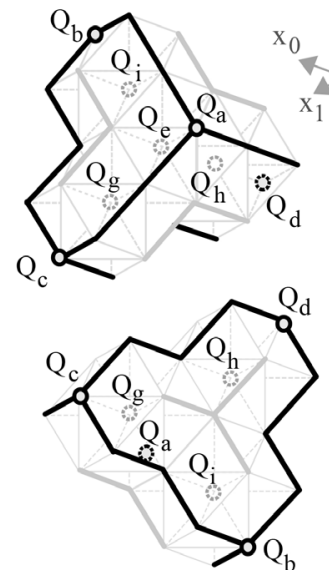

(b)
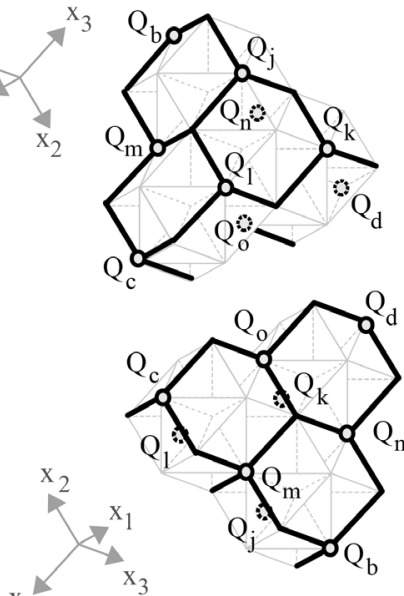

(c)

Figure 11. Self-eclipsed protein molecules: (a) The surface vein $m_{c}\left(w_{s}, i v_{s}\right)$ with respect to $\left(w_{s}, i v_{s}\right) \cdot m_{c}\left(w_{s}, i v_{s}\right)$ is indicated by the black thick polygonal lines, where $\left(w_{s}, i v_{s}\right)=\left(\operatorname{Cone}^{*}\left\{P_{e}\right\}, \operatorname{ICone}^{*}\left\{P_{f}\right\}\right)$; (b) Closed trajectories of $V_{c_{t}\left(w_{s}, i v_{s}\right)}$. Shown above is the top view. Shown below is the bottom view. $m_{t}\left(c_{t}\left(w_{s}, i v_{s}\right)\right)$ is indicated by the black thick polygonal lines. The grey thick polygonal lines are the diagonal edges of type $\mathrm{E}$ tetrahedrons of Figure 9; (c) Closed trajectories of $V_{c_{1}}$, where $c_{1}=$ Cone $\left\{P_{b}, P_{c}, P_{d}, P_{j}, P_{k}, P_{l}, P_{m}, P_{n}, P_{o}\right\} . m_{t}\left(c_{1}\right)$ is indicated by the black thick polygonal lines. Note that all the tetrahedrons are type D. In the figure, $Q_{x}=\pi_{p t}\left(P_{x}\right)$ $(x=a, b, c, \cdots, p)$, where $P_{a}=x_{0}^{2} x_{1}^{0} x_{2}^{2} x_{3}^{2}, P_{b}=x_{0}^{3} x_{1}^{2} x_{2}^{1} x_{3}^{3}, P_{c}=x_{0}^{3} x_{1}^{2} x_{2}^{3} x_{3}^{1}, \quad P_{d}=x_{0}^{1} x_{1}^{2} x_{2}^{3} x_{3}^{3}$, $P_{e}=x_{0}^{1} x_{1}^{0} x_{2}^{1} x_{3}^{1}, \quad P_{f}=x_{0}^{6} x_{1}^{6} x_{2}^{6} x_{3}^{6}, \quad P_{g}=x_{0}^{5} x_{1}^{4} x_{2}^{5} x_{3}^{4}, \quad P_{h}=x_{0}^{4} x_{1}^{4} x_{2}^{5} x_{3}^{5}, \quad P_{i}=x_{0}^{5} x_{1}^{4} x_{2}^{4} x_{3}^{5}$, $P_{j}=x_{0}^{3} x_{1}^{1} x_{2}^{2} x_{3}^{3}, \quad P_{k}=x_{0}^{2} x_{1}^{1} x_{2}^{3} x_{3}^{3}, \quad P_{l}=x_{0}^{3} x_{1}^{1} x_{2}^{3} x_{3}^{2}, \quad P_{m}=x_{0}^{3} x_{1}^{2} x_{2}^{2} x_{3}^{2}, \quad P_{n}=x_{0}^{2} x_{1}^{2} x_{2}^{2} x_{3}^{3}$, $P_{o}=x_{0}^{2} x_{1}^{2} x_{2}^{3} x_{3}^{2}$, and $P_{p}=x_{0}^{5} x_{1}^{6} x_{2}^{5} x_{3}^{5} \quad\left(Q_{p}\right.$ is the diametrically opposite point to $Q_{a}$ ).

$R_{t}\left(c_{1}\right)$ consists of 13 closed trajectories of length six, which sweep the same region as $R_{t}\left(c_{t}\left(w_{s}, i v_{s}\right)\right)$ considered in the proof of Theorem 6 .

On the other hand,

$$
\left\{\begin{array}{l}
\left(w_{c}\left(c_{1}\right), i v_{c}\left(c_{1}\right)\right)=\left(\operatorname{Cone}^{*}\left\{P_{e}\right\}, \text { ICone }^{*}\left\{P_{q}, P_{r}, P_{s}\right\}\right), \\
R_{c}\left(w_{c}\left(c_{1}\right), i v_{c}\left(c_{1}\right)\right)=R_{t}\left(c_{1}\right),
\end{array}\right.
$$

where $P_{q}=x_{0}^{5} x_{1}^{4} x_{2}^{5} x_{3}^{4}, P_{r}=x_{0}^{4} x_{1}^{4} x_{2}^{5} x_{3}^{5}$, and $P_{s}=x_{0}^{5} x_{1}^{4} x_{2}^{4} x_{3}^{5}$.

Then, $c_{t}\left(w_{c}\left(c_{1}\right), i v_{c}\left(c_{1}\right)\right)=c_{1}$ and

$$
\begin{aligned}
\tau_{3}\left(R_{c}\left(w_{c}\left(c_{1}\right), i v_{c}\left(c_{1}\right)\right)\right) & =R_{t}\left(c_{t}\left(w_{c}\left(c_{1}\right), i v_{c}\left(c_{1}\right)\right)\right) \\
& =R_{t}\left(c_{1}\right)=R_{c}\left(w_{c}\left(c_{1}\right), i v_{c}\left(c_{1}\right)\right) .
\end{aligned}
$$

That is, The complex of closed trajectories included in $R_{c}\left(w_{c}\left(c_{1}\right), i v_{c}\left(c_{1}\right)\right)$ is not a self-eclipsed protein molecule.

\section{Systems of Simultaneous Equations for Shape}

Protein molecules interact each other and form an intermediate complex to 
perform their function. In high-throughput proteomics, proteins are characterized using a interaction network between proteins and intermediate protein complexes. Since the function of a protein is primarily determined by the three-dimensional shape, it is the shape of proteins that is characterized by the interaction network.

In this section, we will consider "interaction" between closed trajectories of triangles as a simplified geometrical description of protein interactions. Despite its simplicity, the closed trajectory model of protein interaction gives a novel geometrical interpretation of the difference between direct interactions of two proteins and cooperative interactions of three proteins (such as allosteric regulation).

\subsection{Fusion and Fission of Closed Trajectories of Triangles}

We have seen in the previous sections that vector fields of triangles are associated with three-dimensional tangent cones. Here we will define "fusion and fission" of closed trajectories of triangles using the tangent cone structure. For the sake of simplicity, we only consider the case of flows of triangles.

Let $V_{c_{a}}$ and $V_{c_{b}}$ be two vector fields of triangles induced by three-dimensional tangent cones $c_{a}$ and $c_{b}$ respectively. Then, the vector field $V_{c_{a}}$ can be obtained from the other $V_{c_{b}}$ by "putting unit cubes on" and/or "taking unit cubes from" the tangent cone $c_{b}$. Suppose that the closed trajectories of $V_{c_{a}}$ and $V_{c_{b}}$ sweep the same region, i.e., $R_{t}\left(c_{a}\right)=R_{t}\left(c_{b}\right)$ (See Definition 22). Then, the two vector fields $V_{c_{a}}$ and $V_{c_{b}}$ give two different decompositions of the same region into a set of closed trajectories of triangles.

Definition 77 (Addition of closed trajectories) Addition is defined between sets of all the closed trajectories of vector fields. Given two three-dimensional tangent cones $c_{a}$ and $c_{b}$. Let $\left\{m_{i} \mid 0 \leq i \leq N_{a}\right\}$ and $\left\{n_{j} \mid 0 \leq j \leq N_{b}\right\}$ be the set of all the closed trajectories of the vector fields $V_{c_{a}}$ and $V_{c_{b}}$ respectively, where $N_{a}$ and $N_{b}$ is the numbers of the closed trajectories. Then, addition of closed trajectories of triangles is defined by

$$
\sum_{0 \leq i \leq N_{a}} m_{i}:=\sum_{0 \leq j \leq N_{a}} n_{j} \quad \text { if } R_{t}\left(c_{a}\right)=R_{t}\left(c_{b}\right) .
$$

Example 34 Shown in Figure 12(a) is a flow of triangles consisting of 36 closed trajectories of length six and infinitely many open trajectories of various lengths, where each closed trajectory sweeps a hexagonal region. By putting unit cubes on the associated tangent cone, we obtain another decomposition of the same region into a set of closed trajectories as shown in Figure 12(b). Then, we have

$$
\begin{aligned}
\sum_{0 \leq i \leq 35} x_{i}= & \sum_{0 \leq j \leq 5} m_{j}+\sum_{0 \leq i \leq 3} x_{i}+x_{4}+x_{8}+x_{9}+x_{14}+x_{15}+x_{20} \\
& +x_{21}+x_{22}+x_{26}+x_{27}+x_{28}+x_{31}+\sum_{32 \leq i \leq 35} x_{i} .
\end{aligned}
$$

By removing the common terms from both sides, we obtain

$$
\begin{aligned}
& x_{5}+x_{6}+x_{7}+x_{10}+x_{11}+x_{12}+x_{13}+x_{16}+x_{17} \\
& +x_{18}+x_{19}+x_{23}+x_{24}+x_{25}+x_{29}+x_{30} \\
& =m_{0}+m_{1}+m_{2}+m_{3}+m_{4}+m_{5} .
\end{aligned}
$$



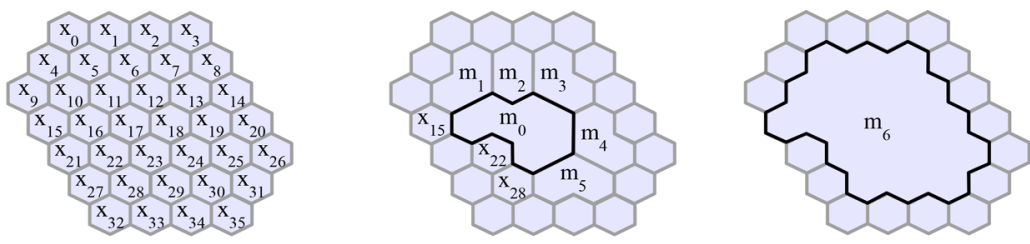

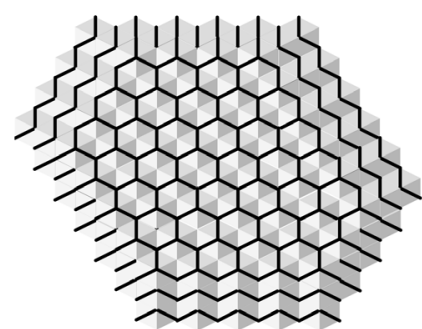

(a)

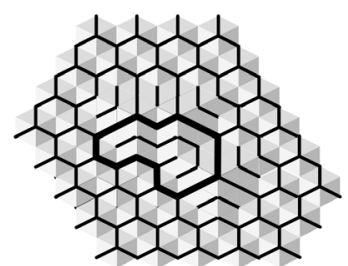

(b)

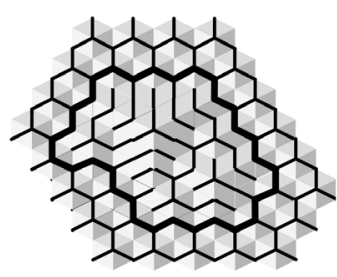

(c)

Figure 12. Addition of closed trajectories: (a) A flow of triangles consisting of 36 closed trajectories of length six. Shown below is the corresponding tangent cone (top view); (b) Closed trajectories of triangles obtained by putting unit cubes on the tangent cone of (a); (c) Closed trajectories of triangles obtained by putting unit cubes on the tangent cone of (b).

Note that $m_{0}$ is the closed trajectory given in Figure 1 . That is, we obtained $m_{0}$ as a result of "fusion and fission" of 16 hexagons.

Example 35 By putting more unit cubes on the tangent cone of Figure 12(b), we obtain another equation

$$
m_{0}+m_{1}+m_{2}+m_{3}+m_{4}+m_{5}+x_{15}+x_{22}+x_{28}=m_{6} .
$$

Then, $m_{0}$ appears as a "factor" of a longer closed trajectory $m_{6}$.

In the above examples, closed trajectories $x_{i}$ (of length six) are given first. Then, $m_{0}$ is obtained as a result of interactions of the $x_{i}$. The challenge we propose is to give a set of equations of $m_{0}$ on variables $x_{i}$ s first, and solve the system of simultaneous equations.

Open Problem 1 (Simultaneous equations for shape) Let $\left\{x_{0}, x_{1}, \cdots, x_{N}\right\}$ be a finite set of closed trajectories of length six, i.e., hexagons. Let $\left\{m_{0}, m_{1}, \cdots, m_{M}\right\}$ be a finite set of closed trajectories of length longer than six. Suppose that we are given a finite set of addition equations with respect to $x_{S}$ and $m_{j} s$.

$$
\begin{aligned}
& f_{k}\left(x_{0}, x_{1}, \cdots, x_{N}, m_{0}, m_{1}, \cdots, m_{M}\right) \\
& =g_{k}\left(x_{0}, x_{1}, \cdots, x_{N}, m_{0}, m_{1}, \cdots, m_{M}\right), \quad(0 \leq k \leq K),
\end{aligned}
$$

where $f_{k}$ and $g_{k} s$ are finite sets of terms separated by addition sign (i.e., addition expressions with coefficients one).

Find three-dimensional tangent cones for the variables $x_{i}$ s that make the addition equations true, where $X_{P} s$ are assigned the closed trajectory induced by the corresponding tangent cone. Then, $m_{i} s$ are obtained as intermediate products of the interactions between $x_{i}$ s.

Since the interaction of closed trajectories is primarily determined by their contours, it is their contours which are characterized by a set of simultaneous equations. In the case of Open Problem 1, a closed trajectory $m_{0}$ is characterized 
using interactions between closed trajectories of length six and other closed trajectories. Therefore, the set of equations is nothing but a specification of the shape of $m_{0}$ if $m_{0}$ is uniquely determined.

\subsection{Allosteric Regulation of Interactions}

Now let us consider the difference between direct interactions of two proteins and cooperative interactions of three proteins (such as allosteric regulation). In our closed trajectory model, allosteric regulation corresponds to the complex of self-eclipsed closed trajectories of triangles (Definition 36). We will start with a brief introduction to allosteric regulation.

\subsubsection{Introduction to Allosteric Regulation}

In biological systems, all proteins bind to other molecules to carry out their functions. For example, enzymes bind to one or more reactant molecules to catalyze chemical reactions in our body. The region on the surface to which other molecules bind is called the active site.

The binding of a molecule at an active site is often controlled by the binding of another molecule at a distant site other than the active site. This type of regulation of protein function is called allosteric regulation. The distant site is called an allosteric site.

Allosteric regulation, which is known as "the second secret of life"', second only to the genetic code [9] [10], is ubiquitous in biological processes. But we still lack general understanding of the mechanisms underlying the coupling between allosteric and active sites [11] [12].

Allosteric regulation is typically triggered by the binding of a small molecule, but also triggered by the binding of another protein. When proteins bind to other molecules or proteins, changes in conformation and/or dynamics occur within the protein. Classically, allosteric regulation was considered to be induced through a change in conformation of the protein. Today, it is believed that allostery can take place through a change in the dynamic fluctuations (i.e., internal motions and vibrations) of the protein even without obvious conformational changes [13] [14] [15].

Currently almost all the drugs modify the actions of proteins by directly binding to their active sites. On the other hand, gaining increasing attention recently in drug discovery is another type of drugs, called allosteric drugs, which bind to the allosteric sites on their target proteins [16] [17] [18]. This is because allosteric drugs have several advantages over traditional drugs, such as higher specificity, fewer side effects, and lower toxicity.

However, allosteric drug discovery is more challenging than traditional drug discovery due to difficulties in identification of allosteric sites, prediction of drug modulatory effects, and others. For example, allosteric sites may have features we are not yet aware of because of our insufficient understanding of how coupling between the active site and the allosteric site occurs.

In the past ten years, various computational approaches have been developed 
for identification and characterization of allosteric sites as the first step in allosteric drug discovery [19] [20] [21]. In a static geometry-based model, protein structures are transformed into residue interaction graphs (RIGs), where amino acid residues are graph nodes and their interactions are the graph edges [22]. Then, allosteric regulation and communication are characterized using a local centrality measure (local closeness) and other newly developed quantifiable measures (binding leverage and leverage coupling). In normal mode analysis (NMA)-based models, proteins are represented by a set of $\mathrm{Ca}$ atoms interconnected by a network of elastic springs. Then, the structural fluctuations of a protein are decomposed into harmonic orthogonal modes and the long-range nature of allosteric communication is often well-described by low-frequency modes. However, Molecular dynamics (MD) remains the standard computational tool for structural analysis when structures are available.

\subsubsection{The SECT Model of Allosteric Regulation}

In this paper, we propose a novel geometrical interpretation of the long-distance regulation of protein interactions (with no conformational change). In particular, we consider how the coupling between active and allosteric sites occurs using the simplified structural description. Analysis of this model has allowed us to characterize a novel geometrical aspect of the structural coupling between active and allosteric sites.

Definition 78 (The SECT model of allosteric regulation) The Self-Eclipsed Closed Trajectory (SECT) model is a simplified geometrical interpretation of protein interactions, where

- Protein molecules correspond to a complex of closed trajectories of triangles,

- Protein interactions are represented as additions of the corresponding complexes of closed trajectories,

- Protein molecules with allosteric sites correspond to a complex of self-eclipsed closed trajectories,

- An allosteric site is the region of the contour eclipsed by the active site.

Note that the SECT model is a purely theoretical model. The SECT model was devised in the process of searching the definition formula of the shape of proteins. In the model, the constraint on the contour and the coupling between two sites are two sides of the same coin as shown in the example below. Roughly speaking, active and allosteric sites are closely tied to each other as an entity and its shadow.

Remark. Recall that (slant) triangles are flowing on the slopes of a "tangent" cone. On the other hand, the contour of a closed trajectory is drawn on the slopes of a "cotangent" cone. Since the "slope inclination" of tangent cones is gentler than that of cotangent cones, the existence of a local trajectory of triangles at one region (active site) of the contour affect the existence of another local trajectory at another region (allosteric site) of the contour.

Example 36 Shown in Figure 13 is the interactions between the three closed trajectories $m_{0}, m_{2}$, and $x_{22}$ of Figure 12(b). The interaction between $m_{0}$ and $x_{22}$ 


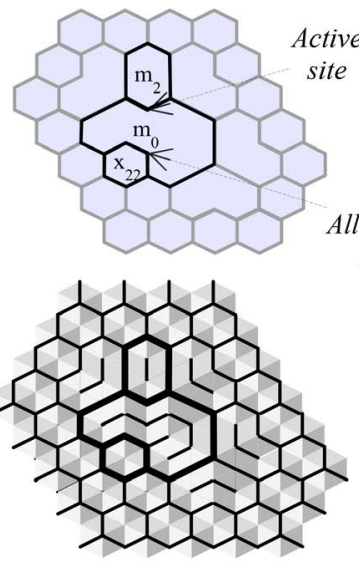

(a)
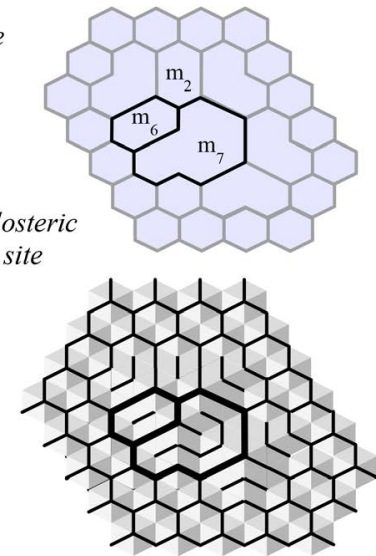

(b)
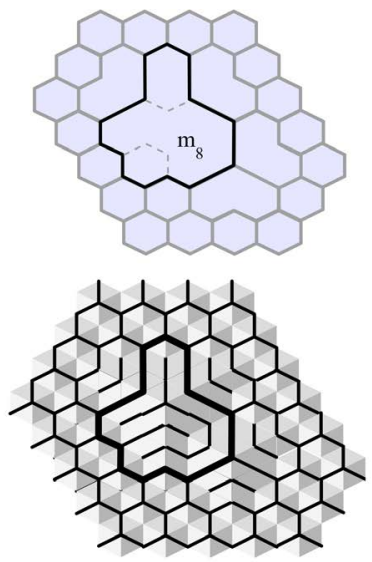

(c)

Figure 13. Allosteric regulation and complexes of self-eclipsed closed trajectories: (a) An active site and an allosteric site of a self-eclipsed closed trajectory $m_{0}$. Shown below is the corresponding three-dimensional tangent cone (top view); (b) Interaction of $m_{0}$ and $x_{22}$, i.e., $m_{0}+x_{22}=m_{6}+m_{7}$; (c) Interaction of $m_{0}, m_{2}$, and $x_{22}$, i.e., $m_{0}+x_{22}+m_{2}=m_{8}$.

is obtained by putting two unit cubes on the tangent cone of (a) (Figure 13(b)). We also obtain the interaction of the three closed trajectories $m_{0}, m_{2}$, and $x_{22}$ by putting one more unit cube on the tangent cone of (b) (Figure 13(c)). However, $m_{0}$ and $m_{2}$ do not interact without the binding of $x_{22}$ because of the overlap of the slopes of the tangent cone, i.e., self-eclipse. That is,

$$
\left\{\begin{array}{l}
m_{0}+x_{22}=m_{6}+m_{7}, \\
m_{0}+m_{2}=m_{0}+m_{2}, \quad\left(\text { no interaction without } x_{22}\right) \\
m_{0}+x_{22}+m_{2}=m_{8} .
\end{array}\right.
$$

In this case, $x_{22}$ activates the interaction between $m_{0}$ and $m_{2}$.

\section{Conclusions}

We have proposed a novel simplified geometrical description of the shape of protein molecules and their interactions. Using the model, we have identified not only global geometrical constraints on the shape of proteins, but also their influence on protein interactions. As an example of the global constraints, a "garlic-bulb like structure" was shown. As an example of their influence on interactions, the structural coupling between active and allosteric sites was considered. In particular, our model gives a novel geometrical interpretation of the long-distance regulation of protein interactions, which could be important for the understanding of the basic mechanisms of allosteric regulation of protein functions.

As for future research questions, we have already proposed an open problem in the text, i.e., the problem of simultaneous equations for shape. Just as the function (i.e., shape) of a protein is determined by its interaction with other molecules, the shape of a complex of closed trajectories may be determined uniquely by its interaction (i.e., fusion and fission) with other closed trajectories. 
Another open problem is about the relationship between the shape of a complex of closed trajectories of tetrahedrons and the triangle flow induced on the surface. How far can we learn about the three-dimensional shape from the two-dimensional surface triangle flow? To answer the question, we should consider a patchwork of locally overlapping three-dimensional cones that cover the surface of the complex because the surface triangle flow has "singular points".

From the viewpoint of computer engineering, development of a "protein description language" will be required if proteins are to be designed automatically. The model of the closed trajectories of tetrahedrons (or $n$-simplices) is expected to give the semantics of the language as in the case of mathematical semantics and the lambda calculus [23].

\section{Conflicts of Interest}

The author declares no conflicts of interest regarding the publication of this paper.

\section{References}

[1] Morikawa, N. (2014) Discrete Differential Geometry of $n$-Simplices and Protein Structure Analysis. Applied Mathematics, 5, 2458-2463. https://doi.org/10.4236/am.2014.516237

[2] Morikawa, N. (2017) Discrete Differential Geometry and the Structural Study of Protein Complexes. Open Journal of Discrete Mathematics, 7, 148-164. https://doi.org/10.4236/ojdm.2017.73014

[3] Saitoh, S., Nakai, T. and Nishikawa, K. (1993) A Geometrical Constraint Approach for Reproducing the Native Backbone Conformation of a Protein. Proteins, 15, 191-204. https://doi.org/10.1002/prot.340150209

[4] Ramachandran, G.N., Ramakrishnan, C. and Sasisekharan, V. (1963) Stereochemistry of Polypeptide Chain Configurations. Journal of Molecular Biology, 7, 95-99. https://doi.org/10.1016/S0022-2836(63)80023-6

[5] Lund, O., Hansen, J., Brunak, S. and Bohr, J. (1996) Relationship between Protein Structure and Geometrical Constraints. Protein Science, 5, 2217-2225.

https://doi.org/10.1002/pro.5560051108

[6] Prasad, B.V. and Schmid, M.F. (2012) Principles of Virus Structural Organization. Advances in Experimental Medicine and Biology, 726, 17-47.

https://doi.org/10.1007/978-1-4614-0980-9_3

[7] Keef, T., Wardman, J.P., Ranson, N.A., Stockley, P.G. and Twarock, R. (2013) Structural Constraints on the Three-Dimensional Geometry of Simple Viruses: Case Studies of a New Predictive Tool. Acta Crystallographica, Section A, A69, 140-150. https://doi.org/10.1107/S0108767312047150

[8] Mannige, R.V. and Brooks 3rd, C.L. (2008) Tilable Nature of Virus Capsids and the Role of Topological Constraints in Natural Capsid Design. Physical Review E, Statistical, Nonlinear, and Soft Matter Physics, 77, Article ID: 051902. https://doi.org/10.1103/PhysRevE.77.051902

[9] Monod, J. (1977) Chance and Necessity: Essay on the Natural Philosophy of Modern Biology. Penguin Books Ltd., London. 
[10] Edelstein, S.J. (2013) Allosteric Interactions after 50 Years. Journal of Molecular Biology, 425, 1391-1395. https://doi.org/10.1016/j.jmb.2013.03.026

[11] Laskowski, R.A., Gerick, F. and Thornton, J.M. (2009) The Structural Basis of Allosteric Regulation in Proteins. FEBS Letters, 583, 1692-1698. https://doi.org/10.1016/j.febslet.2009.03.019

[12] Liu, J. and Nussinov, R. (2016) Allostery: An Overview of Its History, Concepts, Methods, and Applications. PLoS Computational Biology, 12, e1004966. https://doi.org/10.1371/journal.pcbi.1004966

[13] Cooper, A. and Dryden, D.T. (1984) Allostery without Conformational Change. European Biophysics Journal, 11, 103-109. https://doi.org/10.1007/BF00276625

[14] Kalodimos, C.G. (2011) NMR Reveals Novel Mechanisms of Protein Activity Regulation. Protein Science, 20, 773-782. https://doi.org/10.1002/pro.614

[15] Motlagh, H.N., Wrabl, J.O., Li, J. and Hilser, V.J. (2014) The Ensemble Nature of Allostery. Nature, 508, 331-339. https://doi.org/10.1038/nature13001

[16] Nussinov, R. and Tsai, C.J. (2013) Allostery in Disease and in Drug Discovery. Cell, 153, 293-305. https://doi.org/10.1016/j.cell.2013.03.034

[17] Guarnera, E. and Berezovsky, I.N. (2016) Allosteric Sites: Remote Control in Regulation of Protein Activity. Current Opinion in Structural Biology, 37, 1-8. https://doi.org/10.1016/j.sbi.2015.10.004

[18] Huang, W., Nussinov, R. and Zhang, J. (2017) Tools for Allosteric Drug Discovery: Site Identification and Focus Library Design. Methods in Molecular Biology, 1529, 439-446. https://doi.org/10.1007/978-1-4939-6637-0_23

[19] Lu, S., Huang, W. and Zhang, J. (2014) Recent Computational Advances in the Identification of Allosteric Sites in Proteins. Drug Discovery Today, 19, 1595-1600. https://doi.org/10.1016/j.drudis.2014.07.012

[20] Wagner, J.R., Lee, C.T., Durrant, J.D., Malmstrom, R.D., Feher, V.A. and Amaro, R.E. (2016) Emerging Computational Methods for the Rational Discovery of Allosteric Drugs. Chemical Reviews, 116, 6370-6390. https://doi.org/10.1021/acs.chemrev.5b00631

[21] Greener, G.G. and Sternberg, M.J. (2018) Structure-Based Prediction of Protein Allostery. Current Opinion in Structural Biology, 50, 1-8. https://doi.org/10.1016/j.sbi.2017.10.002

[22] Mitternacht, S. and Berezovsky, I.N. (2011) A Geometry-Based Generic Predictor for Catalytic and Allosteric Sites. Protein Engineering Design and Selection, 24, 405-409. https://doi.org/10.1093/protein/gzq115

[23] Scott, D.S. and Strachey, C. (1971) Towards a Mathematical Semantics for Computer Languages, Technical Monograph PRG-6. Programming Research Group, Oxford University Computing Laboratory, Oxford. 


\section{List of Symbols}

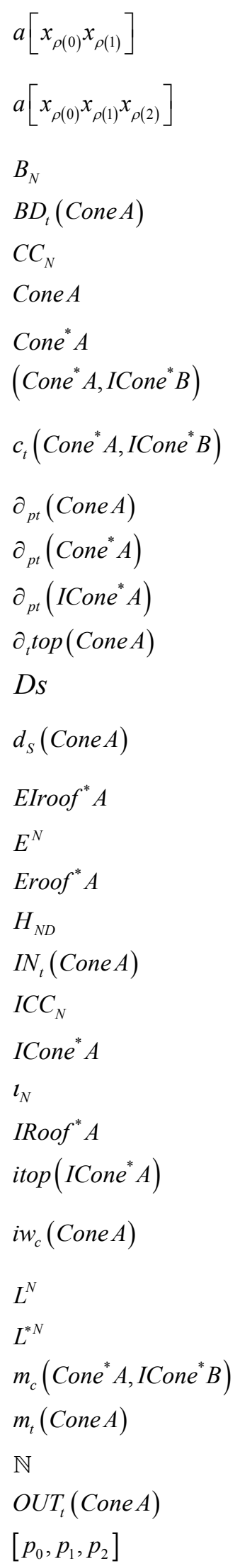

slant triangle defined by three points $a, a x_{\rho(0)}$ and $a x_{\rho(0)} x_{\rho(1)}$ (Def.2) slant tetrahedron defined by four points $a, a x_{\rho(0)}, a x_{\rho(0)} x_{\rho(1)}$ and $\operatorname{ax}_{\rho(0)} x_{\rho(1)} x_{\rho(2)}$ (Def.38)

set of all flat triangles/tetrahedrons (Def.5, Def.41)

set of slant triangles/tetrahedrons of $d_{S}$ (Cone A) (Def.21, Def.57)

set of $N+1$-dimensional tangent cones (Def.33, Def.73)

three/four-dimensional tangent cone (Def.8, Def.44)

three/four-dimensional cotangent cone (Def.14, Def.50)

three/four-dimensional contour/surface pair (Def.26, Def.66)

three/four-dimensional tangent cone associated with $\left(\operatorname{Cone}^{*} A, \operatorname{ICone}^{*} B\right)$

(Def.32, Def.72)

surface lattice points of Cone A (Def.10, Def.46)

surface lattice points of $\operatorname{Cone}^{*} A$ (Def.16, Def.52)

surface lattice points of ICone ${ }^{*} A$ (Def.25, Def.65)

peaks on the boundary of Cone $A$ (Def.9, Def.45)

gradient of slant triangle/tetrahedron $s$ (Def.3, Def.39)

set of all the slant triangles/tetrahedrons on the surface of Cone $A$

(Def.11, Def.47)

four-dimensional extended inverted cotangent cone (Def.64)

$N$-dimensional Eucledean space

four-dimensional extended cotangent roof (Def.60)

hyperplane in $E^{N+1}$ (above Def.5, above Def.41)

set of slant triangles/tetrahedrons of $d_{S}$ (Cone A) (Def.21, Def.57)

set of $N+1$-dimensional tangent cones (Def.33, Def.73)

three/four-dimensional inverted cotangent cone (Def.23, Def.63)

map from $R_{t, N}$ to $R_{c, N}$ (Def.35, Def.75)

three/four-dimensional inverted cotangent roof (Def.24, Def.64)

set of all the top vertices of ICone ${ }^{*} A$ (Def.23, Def.63)

three/four-dimensional inverted cotangent roof associated with Cone $A$

(Def.31, Def.70)

$N$-dimensional standard lattice (Def.1, Def.37)

$N$-dimensional conjugate lattice (Def.13, Def.49)

contour/surface vein with respect to $\left(\right.$ Cone $^{*}$ A, ICone $\left.{ }^{*} B\right)$ (Def.29, Def.69)

contour/surface vein with respect to ConeA (Def.20, Def.56)

set of all natural numbers

set of slant triangles/tetrahedrons of $d_{S}$ (Cone A) (Def.21, Def.57)

convex hull of points $p_{0}, p_{1}$ and $p_{2}$ (above Def.2) 


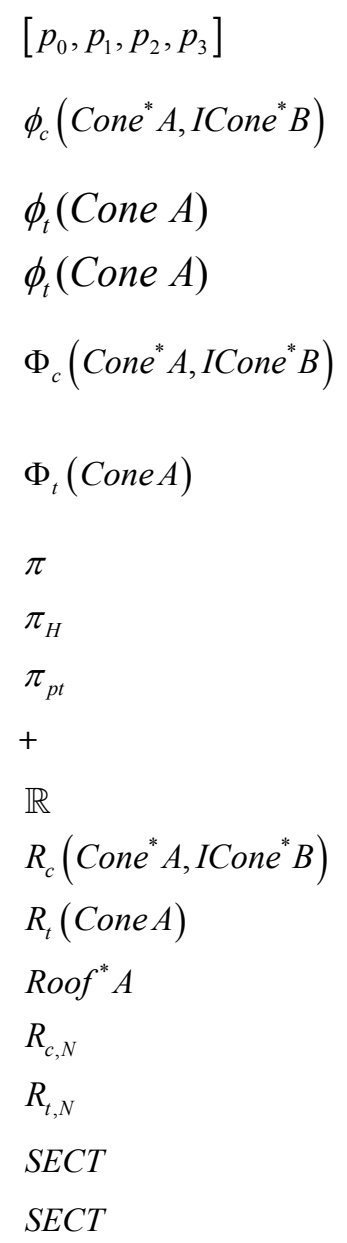

Self-eclipsed contour/surface pair Self-eclipsed protein molecule $S_{N}$ $s_{D D}$ convex hull of points $p_{0}, p_{1}, p_{2}$ and $p_{3}$ (above Def.38)

contour/surface vertices with respect to $\left(\right.$ Cone $^{*} A$, ICone $\left.^{*} B\right)$

(Def.27, Def.67)

contour/surface vertices with respect to ConeA (Def.18, Def.54)

extended surface vertices with respect to ConeA (Def.62)

polygonal line (or set of the polygonal lines) obtained by joining the adjacent $L^{* N}$ lattice points of $\phi_{c}\left(\right.$ Cone $^{*} A$, ICone $\left.^{*} B\right)$ (Def.28, Def.68)

polygonal line (or set of the polygonal lines) obtained by joining the adjacent $L^{* N}$ lattice points of $\phi_{t}($ Cone $A)$ (Def.19, Def.55)

projection of $T B_{N}$ onto $B_{N}$ (Def.7, Def.43)

projection of $S_{N}$ onto $H_{N D}$ (Def.5, Def.41)

projection of $E^{N+1}$ onto $H_{N D}$ (above Def.5, above Def.41)

addition of closed trajectories (Def.77)

set of all real numbers

region of $H_{N D}$ (Def.30, Def.71)

region of $H_{N D}$ (Def.22, Def.58)

three/four-dimensional cotangent roof (Def.15, Def.51)

region of $H_{N D}$ (Def.34, Def.74)

region of $H_{N D}$ (Def.34, Def.74)

self-eclipsed closed trajectory complexes (Def.36)

model of allosteric regulation (Def.78)

(Def.36, Def.76)

(Def.76)

set of all slant triangles/tetrahedrons (Def.2, Def.38)

slant triangle/tetrahedron adjacent to $s$ (Def.4, Def.40)

slant triangle/tetrahedron adjacent to $s$ (Def.4, Def.40)

slant triangle/tetrahedron adjacent to $s$ (Def.4, Def.40)

slant triangle/tetrahedron adjacent to $s$ (Def.4, Def.40)

stand of $a($ Def.59)

stand of $A$ (Def.59)

group of all permutations of the $N$-elements set $\{0,1, \cdots, N-1\}$

map from $R_{c, N}$ to $R_{t, N}$ (Def.35, Def.75)

tangent space on $B_{N}$ (Def.7, Def.43)

set of $N+1$-dimensional tangent cones (Def.33, Def.73)

set of all the top vertices of Cone A (Def.8, Def.44)

vector field induce by Cone $A$ on $B_{N}$ (Def.12, Def.48)

three/four-dimensional cotangent roof associated with Cone $A$

(Def.17, Def.53) 
$w_{c}($ Cone $A)$

$x_{0}^{l} x_{1}^{m} x_{2}^{n}$

$x_{0}^{l} x_{1}^{m} x_{2}^{n} x_{3}^{k}$

$\mathbb{Z}$ four-dimensional extended cotangent roof associated with Cone $A$ (Def.61)

point or vector $(l, m, n)$ in $E^{3}$

point or vector $(l, m, n, k)$ in $E^{4}$

set of all integers 